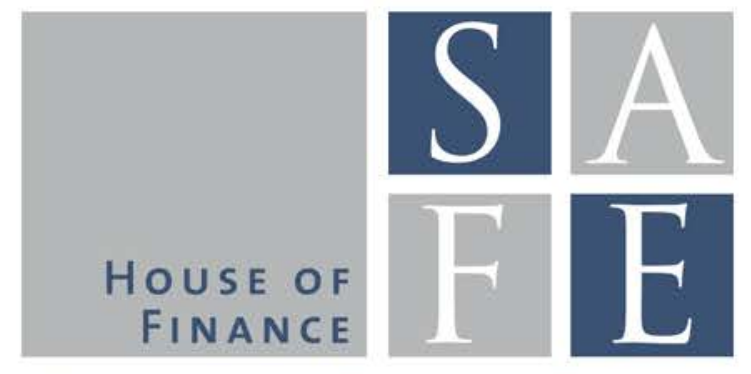

Working PAPER SERIES

Daniel Harenberg - Alexander Ludwig

\title{
Social Security in an Analytically Tractable Overlapping Generations Model with Aggregate and Idiosyncratic Risk
}

SAFE Working Paper No. 71

SAFE I Sustainable Architecture for Finance in Europe A cooperation of the Center for Financial Studies and Goethe University Frankfurt 


\section{Non-Technical Summary}

Many countries operate large social security systems. Social security can increase economic efficiency and provide insurance against household risks for which no private markets exist. However, social security systems also impose costs by distorting prices and decisions. The question arises whether the benefits of social security outweigh the costs.

To study the welfare consequences of introducing a social security system this paper develops an analytically tractable model with two overlapping generations where households face many different sources of risks over their life-cycle. Key risks for the analysis of social security are idiosyncratic risks - e.g., the risk of unemployment - and aggregate risks - i.e., the risk of aggregate productivity losses which leads to reductions of wages and asset returns. Our analysis on the welfare effects of social security in light of these risks differs from the previous literature in that, thus far, prior studies have only considered models with one type of risk in isolation. One strand of the literature has examined social security when only aggregate risk is present. The other strand has only considered idiosyncratic risk.

In our model we find that the two risks interact in multiple ways and influence the welfare effects of introducing a social security system. As a result, we show that these effects are less strong on the welfare costs of crowding out than on the welfare gains from insurance. Hence, the interactions of risks increase the total welfare benefits of social security.

The findings of this paper must be investigated in a more realistic quantitative model which is suitably calibrated. This is done in our companion paper, Harenberg and Ludwig (2014). There we document that indeed the interactions of risks overturn conventional findings on the welfare effects of social security and we conclude that the introduction of a minimum flat pension is welfare improving once all household risks are appropriately taken into account. 


\title{
Social Security in an Analytically Tractable Overlapping Generations Model with Aggregate and Idiosyncratic Risk*
}

\author{
Daniel Harenberg ${ }^{\dagger a}$ and Alexander Ludwig ${ }^{\ddagger b}$ \\ ${ }^{\mathrm{a}} \mathrm{ETH}$, Zürich

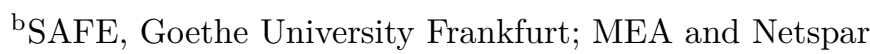

This Version: September 22, 2014

\begin{abstract}
When markets are incomplete, social security can partially insure against idiosyncratic and aggregate risks. We incorporate both risks into an analytically tractable model with two overlapping generations and demonstrate that they interact over the life-cycle. The interactions appear even though the two risks are orthogonal and they amplify the welfare consequences of introducing social security. On the one hand, the interactions increase the welfare benefits from insurance. On the other hand, they can in- or decrease the welfare costs from crowding out of capital formation. This ambiguous effect on crowding out means that the net effect of these two channels is positive, hence the interactions of risks increase the total welfare benefits of social security.
\end{abstract}

JEL classification: C68; E27; E62; G12; H55

Keywords: social security; idiosyncratic risk; aggregate risk; welfare; insurance; crowding out

*This is a companion paper to our quantitative work in Harenberg and Ludwig (2014). Daniel Harenberg gratefully acknowledges financial support of the Sonderforschungsbereich SFB 884 (German National Research Foundation), and of Swiss Re. Alex Ludwig gratefully acknowledges research support from the Research Center SAFE, funded by the State of Hessen initiative for research LOEWE and financial support by the German National Research Foundation under SPP 1578.

$\dagger$ ETH, Zürich; Zürichbergstrasse 18; 8092 Zürich; Email: dharenberg@ethz.ch.

${ }^{\ddagger}$ SAFE, Goethe University Frankfurt; Grüneburgplatz 1; 60323 Frankfurt am Main; Germany; E-mail: ludwig@safe.uni-frankfurt.de. 


\section{Introduction}

Almost all industrialized countries have large public social security systems with sizeable pay-as-you-go (PAYG) components. In such systems payments to current pensioners are financed by taxing current workers. Social security can hence improve intergenerational risk sharing by pooling aggregate risks across generations. In addition, most systems have some form of redistributional component. Hence, social security can also insure against idiosyncratic earnings risks for which private markets do not exist and for which other government transfers only provide partial insurance. However, these systems are financed by distortionary taxes. The question arises whether the benefits from insurance outweigh the costs of distortionary taxation.

The present paper demonstrates that the benefits from insurance have been underestimated in the previous literature because either aggregate or idiosyncratic risks have been studied in isolation. Such a segregated view provides only an incomplete picture and, crucially, misses important interactions between the risks. These interactions typically arise in economic models even if the risks are orthogonal by construction. The reason is that they multiply each other in the budget constraint, either explicitly or implicitly, as explained below. Also, they often interact in the utility function, where one of the risks can often be thought of as a background risk in the sense of Gollier and Pratt (1996) or Franke, Schlesinger, and Stapleton (2006). It is unknown how such interactions affect the benefits from insurance and the cost of distortionary taxation. This paper aims to fill that gap.

The risks interact in the budget constraint whenever they multiply each other. For example, both an idiosyncratic wage shock and an aggregate wage shock enter as a product to the aggregate wage. From an ex-ante perspective, the variance of the aggregate wage will be larger than the sum of the individual variances. ${ }^{1}$ As a consequence, the variance of consumption will become larger from an ex-ante perspective, which in a life-cycle model will increase the value of social security. There is a second multiplicative interaction, which is more interesting, because it is implicit. A household's asset position contains the history of his past shocks, in particular of past idiosyncratic shocks. Part of the assets are saved for next period, and are consequently multiplied with asset returns. If asset returns are stochastic, then implicitly two stochastic variables multiply, the past idiosyncratic shocks and the current return shock. Analogous to before, this increases

\footnotetext{
${ }^{1}$ See Goodman (1960) for an exact formula of the variance of the product of independent stochastic variables.
} 
the variance of the final asset position from an ex-ante perspective by more than the sum of the individual variances. We demonstrate this analytically and show how these interactions increase the value of social security. It is important to stress that the effects are non-negligible because retirement savings decisions take place over long horizons and the interactions accumulate over the life-cycle. Accordingly, we call them life-cycle interactions (LCI).

In addition to the interactions through the budget constraint, the risks interact through the utility function. This idea was first formalized by Gollier and Pratt (1996) who show that in the presence of an independent background risk, households will behave towards a market risk as if they were more more risk-averse than they are. The original background risk was additive, e.g., labor income (the background risk) plus asset income (the market risk). Franke, Schlesinger, and Stapleton (2011) extend this by a multiplicative background risk, which means that the market risk is multiplied with an independent risk. The present paper features a situation as in Franke, Schlesinger, and Stapleton (2011), i.e., with both additive and multiplicative background risk. In contrast to that literature, our model is fully dynamic. As a consequence, the background risks will turn out to be increased by the LCI explained in the previous paragraph.

The model we develop is able isolate all these channels and show their effect on social security analytically. The entire analysis is conducted in a standard overlapping generations (OLG) model with incomplete markets. To maintain analytical tractability, we assume that a household lives for two periods, so that at each point in time, two generations are simultaneously alive. In the first period of life, households earn labor income, which is subject to an aggregate and an idiosyncratic productivity shock. Out of this labor income, they can consume and save. There is a single asset whose return is stochastic, which represents a second aggregate risk. In the second period of life, households eventually retire and receive pension income. Social security is a pure pay-as-you-go (PAYG) system with defined contributions and a lump-sum pension. Our thought experiment considers the introduction of a marginal social security system. This is evaluated using an ex-ante Utilitarian welfare criterion. A crucial assumption maintained throughout is that all shocks are mutually orthogonal, i.e., they are statistically independent of each other. So the interactions do not arise by construction.

Our first set of results looks at insurance provided by social security, and how it is affected when two risks are present. We find that all channels outlined above increase the value of social security in welfare terms. The reason has been already hinted at: LCI increases the variance of retirement consumption, and social security reduces it, which 
from an ex-ante perspective is valuable. At the same time, the presence of background risks, be they multiplicative or additive, effectively increases the risk aversion of households, which implies that they value insurance more. For this set of results we consider only a partial equilibrium of the model, and we assume that households only consume in the second period of life. ${ }^{2}$ For the result including the background risk, we need to subdivide the second period of life into two sub-periods: during the first households work and during the second households are retired.

Our second set of results characterizes the general equilibrium of the economy. In general equilibrium, there is a representative firm with a standard neo-classical production function. Households now have logarithmic utility and consume in both periods of life. We keep the sub-period structure from before. This allows us to jointly evaluate the insurance value of social security and the distortionary effects of the social security contribution rate, maintaining closed form solutions. A higher contribution rate distorts the savings decision and therefore leads to crowding out of aggregate capital with the corresponding welfare consequences. The central result here is that this crowding out is determined by two opposing forces. The interactions enter both of these forces so that it is ambiguous whether they amplify or mitigate the crowding out. This is of importance because for the insurance of social security, the interactions unambiguously increase it. Thus, interactions will generally have a net positive marginal effect on the value of social security.

Essentially, the general equilibrium model can be seen as an extension of the standard Diamond (1965) model with aggregate and idiosyncratic risk. The setup shares similarities with Huffman (1987) with three differences: First, we extend his work by taking into account idiosyncratic risk. Second, we do not only consider positive labor (or endowment) income in the first period of life but rather have two periods with positive labor income. Third, we stick to a two period structure while Huffman (1987) has many periods.

The insight that social security insures against aggregate risks goes back to Diamond (1977) and Merton (1983). Building on this work, Shiller (1999) and Bohn (2001, 2009) show that social security can reduce consumption risk of all generations by pooling labor income and capital income risks across generations if labor income and capital returns are imperfectly correlated.

There is a large quantitative literature building on these insights that aims at quantifying the welfare effects of social security. E.g., Krüger and Kübler (2006), Ludwig and

\footnotetext{
${ }^{2}$ This assumption is also made in very similar contexts by Gordon and Varian (1988), Ball and Mankiw (2007), Matsen and Thogersen (2004), Krüger and Kübler (2006), among others.
} 
Reiter (2010), Olovsson (2010) and Hasanhodzic and Kotlikoff (2013) and others have quantitative models with only aggregate risk whereas Conesa and Krüger (1999), Imrohoroglu, Imrohoroglu, and Joines (1995, 1998), Huggett and Ventura (1999) and Storesletten, Telmer, and Yaron (1999) and many others study insurance through social security against idiosyncratic risk. Our companion paper (Harenberg and Ludwig 2014) combines these two strands of the quantitative literature by developing a quantitative model featuring both risks. There we show that all interactions taken together make up for roughly two thirds of the overall welfare benefits of social security.

As mentioned before, our work also relates to the theoretical literature on background risk, in particular, Gollier and Pratt (1996), Franke, Schlesinger, and Stapleton (2006), and Franke, Schlesinger, and Stapleton (2011). This literature asks how households react with their exposure to one risk when idiosyncratic risk is added, primarily studying portfolio choice problems. As social security can be interpreted as an implicit asset, the decision problem in this paper is similar. However, three differences stand out. First, in our setup, a social planner chooses to implement social security. Hence, the implicit portfolio choice is not made by the household. Second, in any period, social security reduces exposure to wage risk, i.e., reducing jointly the exposure to background risk (idiosyncratic wage risk) and aggregate risk (aggregate wage risk). Third, the literature on background risk largely looks at stylized static examples (with some underlying dynamic motivation). In our structure, the dynamics are made explicit which we emphasize by referring to interactions as life-cycle interactions.

Finally, our work relates to the literature on the welfare costs of aggregate fluctuations initiated by Lucas (1978). De Santis (2007) and Krebs (2007) argue that interactions between idiosyncratic and aggregate risk can increase these costs substantially.

Our analysis proceeds as follows. Section 2 presents the model which is analyzed in Section 3. Section 4 provides a numerical illustration. Section 5 concludes. All proofs are relegated to a Appendix A. Supplementary Appendix ?? - available on our webpagescontains additional results.

\section{The Model}

\subsection{Time and Population}

Time is discrete. Periods in our model are denoted by $t=-\infty, \ldots, 0,1, \ldots, \infty$. In each period, two generations - the young, indexed by $j=1$, and the old, indexed by $j=2$-are 
simultaneously alive. Each generation consists of a continuum of households. Without loss of generality we consider a stationary population.

In our setup, a PAYG pension system would not provide insurance against the risk of longevity even when annuity markets are missing as long as accidental bequests are redistributed, as was shown by Caliendo, Guo, and Hosseini (2013). We therefore do not model survival risk which would, in any case, lead us on a sidetrack. Denoting the period $t$ young population by $N_{1, t}$ and the old by $N_{2, t}$ we accordingly have that $N_{2, t}=N_{1, t}$.

As there is idiosyncratic risk to labor income, we further distinguish by types and denote by $N_{i, j, t}$ the number of households of type $i$ of age $j$ alive in period $t$. We normalize the population of age $j$ to unity, hence $1=N_{t, j}=\int N_{i, j, t} d i$.

\subsection{Households}

A household has preferences over consumption in two periods. In both periods of life, households experience an idiosyncratic productivity shock. ${ }^{3}$ The expected utility function of a household born of type $i$ in period $t$ is given by

$$
U_{t}=(1-\tilde{\beta}) u\left(c_{i, 1, t}\right)+\tilde{\beta} \mathbb{E}_{t}\left[u\left(c_{i, 2, t+1}\right)\right]
$$

where the per period Bernoulli utility function $u$ is (weakly) increasing and concave, i.e., $u^{\prime} \geq 0, u^{\prime \prime}<0$. The factor $\tilde{\beta} \leq 1$ determines the relative weight on first versus second period consumption, and for $\tilde{\beta} \neq 1, \beta \equiv \frac{\tilde{\beta}}{1-\tilde{\beta}}$ is the discount factor. In our notation, we make explicit that households form expectations conditional on the information at their date of birth and therefore denote the expectations operator $\mathbb{E}$ with subscript $t$. As these expectations are formed at the beginning of period $t$, realizations of shocks in period $t$ are in the information set.

We assume that the per period utility function $u$ is CRRA with coefficient of relative risk aversion $\theta$ :

$$
u\left(c_{i, j, t}\right)= \begin{cases}\frac{c_{i, j, t}^{1-\theta}}{1-\theta} & \text { for } \theta \neq 1 \\ \ln \left(c_{i, j, t}\right) & \text { for } \theta=1 .\end{cases}
$$

Households work full time in the first period. For the second period of life, we follow Auerbach and Hassett (2007), Ludwig and Vogel (2009), and others and consider

\footnotetext{
${ }^{3}$ Our analysis below considers restricted versions of the model in which the idiosyncratic shock to productivity either hits in the first or in the second period of life.
} 
a subperiod structure. In the first subperiod - which is of relative length $\lambda \in[0,1)$ households work. We also refer to $\lambda$ as labor productivity in the second period. In the second subperiod - of length $1-\lambda$-households are retired and receive a pension income $b_{t} \geq 0$. The subperiod structure is convenient for analytical reasons. Combined with idiosyncratic income shocks in the second period it enables us to model precautionary savings together with retirement without having to introduce a three-generations structure. This preserves simple first-order difference equations in our characterization of equilibrium dynamics of the economy. ${ }^{4}$

The budget constraints in the two periods are accordingly given by

$$
\begin{aligned}
c_{i, 1, t}+a_{i, 2, t+1} & =(1-\tau) \eta_{i, 1, t} w_{t} \\
c_{i, 2, t+1} & \leq a_{i, 2, t+1}\left(1+r_{t+1}\right)+\lambda \eta_{i, 2, t+1} w_{t+1}(1-\tau)+(1-\lambda) b_{t+1},
\end{aligned}
$$

where $\eta_{i, j, t}$ is the age- $j$, period- $t$ idiosyncratic shock to wages, and $a_{i, 2, t+1}$ denotes savings of a young household, which equal his asset position at the beginning of the following period. Finally, $\tau$ is the (constant) social security contribution rate.

\subsection{Government}

The government organizes a PAYG financed social security system. Pension benefits are lump-sum. Therefore, idiosyncratic wage risks are insured through social security. In each period, the mass of workers who earn aggregate gross wages $w_{t}$ is $L=1+\lambda$. The mass of pensioners is $1-\lambda$. The social security budget constraint therefore writes as

$$
b_{t}(1-\lambda)=\tau w_{t}(1+\lambda) \quad \Leftrightarrow \quad b_{t}=\tau w_{t} \frac{1+\lambda}{1-\lambda} .
$$

\section{$2.4 \quad$ Firms}

To close the model in general equilibrium, we add a firm sector. We assume a rental market setup up with a static optimization problem. Firms maximize profits operating a neo-classical production function. Let profits of the firm be

$$
\Pi=\zeta_{t} F\left(K_{t}, \Upsilon_{t} L\right)-\left(\bar{\delta}+r_{t}\right) \varrho_{t}^{-1} K_{t}-w_{t} L
$$

\footnotetext{
${ }^{4}$ In the Supplementary Appendix ??, we show that a three-generations model would yield very similar results.
} 
where $\zeta_{t}$ is a technology shock and $L$ is total labor which equals $L=1+\lambda$. The technology level, $\Upsilon_{t}$, grows at an exogenous rate $g, \Upsilon_{t}=(1+g) \Upsilon_{t-1}$, for a given $\Upsilon_{0}$. Throughout we assume full depreciation, hence $\bar{\delta}=1$. The variable $\varrho_{t}$ represents an exogenous shock to the unit user costs of capital. We add this non-standard element in order to model additional shocks to the rate of return to capital. These shocks are multiplicative in the user costs to capital for analytical reasons. Production is Cobb-Douglas with capital elasticity $\alpha$,

$$
F\left(K_{t}, \Upsilon_{t} L\right)=K_{t}^{\alpha}\left(\Upsilon_{t} L\right)^{1-\alpha}
$$

Let $k_{t}=\frac{K_{t}}{\Upsilon_{t} L}=\frac{K_{t}}{\Upsilon_{t}(1+\lambda)}$ be the capital intensity, i.e., the capital stock per efficient unit of labor. Then, the firm's first-order conditions are

$$
\begin{aligned}
R_{t}=1+r_{t} & =\alpha k_{t}^{\alpha-1} \zeta_{t} \varrho_{t}=\bar{R}_{t} \zeta_{t} \varrho_{t} \\
w_{t} & =(1-\alpha) \Upsilon_{t} k_{t}^{\alpha} \zeta_{t}=\bar{w}_{t} \zeta_{t},
\end{aligned}
$$

where $\bar{R}_{t}$ denotes the non-stochastic component of the gross return and, likewise, $\bar{w}_{t}$ the non-stochastic component of the per capita wage. Equation (4a) reveals that $\varrho_{t}$ is simply a shock to the gross return on savings, since it does not affect wages.

\subsection{Social Welfare}

We take an ex-ante Rawlsian perspective and specify the social welfare function (SWF) of a cohort born in period $t$ as the unconditional expected utility of being born into period $t$ :

$$
S W F_{t} \equiv \mathbb{E} U_{t}=\mathbb{E}\left[(1-\tilde{\beta}) u\left(c_{i, 1, t}\right)+\tilde{\beta} u\left(c_{i, 2, t+1}\right)\right]
$$

When evaluating the consequences of a reform, we transform this $S W F_{t}$ to a consumption equivalent variation. The consumption equivalent variation $(\mathrm{CEV})$ is the percent increase in consumption required in the pre-reform periods $-\infty, \ldots, 0$ to achieve the same level of ex-ante utility as in the post reform period $t \geq 1$. Denoting the CEV by $g_{c}$, it follows from the utility function (1) that

$$
g_{c t}= \begin{cases}\left(\frac{S W F_{t}}{S W F_{0}}\right)^{\frac{1}{1-\theta}}-1 & \text { for } \theta \neq 1 \\ \exp \left(S W F_{t}-S W F_{0}\right)-1 & \text { for } \theta=1\end{cases}
$$




\subsection{Thought Experiment}

We consider a marginal introduction of social security and investigate how social welfare as defined in Subsection 2.5 is affected by such a policy reform. The policy reform is announced and implemented in period 1. All periods prior to period 1 are pre-reform periods where the economy is in an initial stationary equilibrium.

Our analytical results focus on a comparison of social welfare and sources for welfare gains and losses in two long-run equilibria. To assess the welfare consequences, we accordingly compute $g_{c_{\infty}}$. A cautionary note is in order here. By ignoring transitional dynamics we exaggerate the welfare losses of crowding experienced by generations born in period 0 and during the transition. This is so because the gains from insurance of a reform materialize on impact whereas the complete losses from crowding out only occur in the limit when the new steady state is reached.

\subsection{Stochastic Processes}

To simplify the analysis we assume that both $\zeta_{t}$ and $\varrho_{t}$ are not serially correlated. Despite the observed positive serial correlation of wages and asset returns in annual data, this assumption can be justified on the grounds of the long factual periodicity of each period in a two-period OLG model which is about 30 to 40 years. We also assume that $\zeta_{t}$ and $\varrho_{t}$ are statistically independent so that dependence of return and wage shocks is only reflected through $\zeta_{t}$. The idiosyncratic shocks $\eta_{i, j, t}$ are not correlated with either of the two aggregate shocks. Our key argument below will be to show that the welfare effects of risks interact although they are orthogonal by construction.

Assumption 1. a) Support bounded from below: $\zeta_{t}>0, \varrho_{t}>0$ and $\eta_{i, j, t}>0$ for all $i, j, t$.

b) Means: $\mathbb{E} \zeta_{t}=\mathbb{E} \varrho_{t}=\mathbb{E} \eta_{i, j, t}=1$, for all $i, j, t$.

c) Statistical independence of $\left(\zeta_{t+1}, \zeta_{t}\right)$ and $\left(\varrho_{t+1}, \varrho_{t}\right)$. Therefore: $\mathbb{E}\left(\zeta_{t+1} \zeta_{t}\right)=\mathbb{E} \zeta_{t+1} \mathbb{E} \zeta_{t}$ for all $t$ and, correspondingly, $\mathbb{E}\left(\varrho_{t+1} \varrho_{t}\right)=\mathbb{E} \varrho_{t+1} \mathbb{E} \varrho_{t}$ for all $t$.

d) Statistical independence of $\left(\zeta_{t}, \varrho_{t}\right)$. Therefore: $\mathbb{E}\left(\zeta_{t} \varrho_{t}\right)=\mathbb{E} \zeta_{t} \mathbb{E} \varrho_{t}$ for all $t$.

e) Statistical independence of $\left(\zeta_{t}, \eta_{i, j, t}\right)$. Therefore: $\mathbb{E}\left(\eta_{i, j, t} \zeta_{t}\right)=\mathbb{E} \eta_{i, j, t} \mathbb{E} \zeta_{t}$ for all $i, j, t$.

f) Statistical independence of $\left(\varrho_{t}, \eta_{i, j, t}\right)$. Therefore: $\mathbb{E}\left(\eta_{i, j, t} \varrho_{t}\right)=\mathbb{E} \eta_{i, j, t} \mathbb{E} \varrho_{t}$ for all $i, t$. 


\section{Analysis}

\subsection{Idiosyncratic Risk Early in the Life-Cycle}

We start by looking at a degenerate version of our model in partial equilibrium in which households only care for the second period $(\tilde{\beta}=1)$ and labor productivity in this second period is zero $(\lambda=0)$.

Assumption 2. Let $\tilde{\beta}=1$ and $\lambda=0$.

Due to the partial equilibrium setting, wages and returns are completely exogenous. This allows us to specify directly the stochastic processes driving them. In particular, it allows us to model wages and returns as uncorrelated. We do this, because investigating how such a correlation affects our conclusions would lead us on a sidetrack. Furthermore, as argued by Harenberg, Ludwig, and Maus (2013), a two-generations model is inadequate to fully address the impact of this correlation on the welfare of social security. ${ }^{5}$ Whereas in general equilibrium, both shocks $\zeta_{t}$ and $\rho_{t}$ affect returns, we now assume that there is a separate return shock $\tilde{\varrho}_{t}$ which is independent of $\zeta_{t}$. Thus, our partial equilibrium can be summarized in the following assumption.

Assumption 3. Let $k_{t}=\bar{k}$ given, hence $w_{t}=\bar{w}_{t} \zeta_{t}=\bar{w}_{t-1}(1+g) \zeta_{t}$ and $R_{t}=\bar{R} \tilde{\varrho}_{t}$, where $\tilde{\varrho}_{t}$ has the stochastic properties of $\varrho_{t}$ from Assumption 1.

Since preferences display non-satiation, Assumption 2 implies $c_{i, 1, t}=0$ and $a_{i, 2, t+1}=$ $(1-\tau) \eta_{i, 1, t} \bar{w} \zeta_{t}$. From equations $(2 \mathrm{a}, 2 \mathrm{~b})$ and $(3)$, we accordingly get that retirement consumption is

$$
c_{i, 2, t+1}=\bar{w}_{t}\left(\eta_{i, 1, t} \zeta_{t} \bar{R} \tilde{\varrho}_{t+1}+\tau\left((1+g) \zeta_{t+1}-\eta_{i, 1, t} \zeta_{t} \bar{R} \tilde{\varrho}_{t+1}\right)\right) .
$$

To interpret this, let's first look at a situation where $\tau=0$ and, without loss of generality,

\footnotetext{
${ }^{5}$ Standard analyses, e.g., by Matsen and Thogersen (2004) and Krüger and Kübler (2006), focus on the "hedge view" according to which the welfare benefits of a PAYG social security system are higher the less aggregate wages and returns are correlated. However, this is only true in a two-generations model. If a third generation is added, a counter-veiling force appears. This is emphasized in Harenberg, Ludwig, and Maus (2013). They show that if households sufficiently discount the future, then a larger correlation may increase the welfare benefits of social security. This happens, because now social security reduces the variance of current consumption while increasing the variance of future consumption, which under sufficient discounting increases household utility.
} 
normalize $\bar{w}_{t}=1 .^{6}$ Retirement consumption can then be written as

$$
c_{i, 2, t+1}=\eta_{i, 1, t} \zeta_{t} \tilde{\varrho}_{t+1} \bar{R}
$$

This is formally equivalent to a situation with multiplicative background risk studied in Franke, Schlesinger, and Stapleton (2006).

With regard to a marginal introduction of social security we then have the following result:

Proposition 1. Under Assumptions 1-3, a marginal introduction of social security increases social welfare iff

$$
A_{p e, m b r} \equiv \frac{1+g}{\bar{R}} \frac{\mathbb{E}\left[\frac{1}{\tilde{\varrho}_{t+1}^{\theta}}\right] \mathbb{E}\left[\frac{1}{\zeta_{t}^{\theta}}\right] \mathbb{E}\left[\frac{1}{\eta_{i, 1, t}^{\theta}}\right]}{\mathbb{E}\left[\tilde{\varrho}_{t+1}^{1-\theta}\right] \mathbb{E}\left[\zeta_{t}^{1-\theta}\right] \mathbb{E}\left[\eta_{i, 1, t}^{1-\theta}\right]}-1>0
$$

Proof. See Appendix A.

Observe that term $\frac{1+g}{R}$ in equation (7) reflects the well-known trade-off between an implicit investment in social security and an explicit investment in a risk-free asset. It is the standard Aaron condition (Aaron 1966), which in our context says that in a risk-free environment, an introduction of social security is welfare increasing if and only if $\frac{1+g}{\bar{R}}>1$. The other term in equation (7) is a risk adjustment which scales up the implicit return of social security, $1+g$. The proposition states that, if there is sufficient risk, then the introduction of social security may be welfare improving even when the deterministic version of the economy has $\bar{R}>1+g$. We denote the entire expression as term $A_{p e, m b r}$, where subscript pe stands in for "partial equilibrium" - to distinguish it from the general equilibrium results below - and subscript $m b r$ for multiplicative background risk.

To further interpret the risk adjustment in term $A_{p e, m b r}$, we next assume that all stochastic variables are jointly distributed as log-normal.

Assumption 4. Joint log-normality: $\eta_{i, 1, t}, \zeta_{t}, \zeta_{t+1}, \tilde{\varrho}_{t+1}$ are jointly distributed as lognormal with parameters $\mu_{\ln \eta}, \mu_{\ln \zeta}, \mu_{\ln \tilde{\varrho}}, \sigma_{\ln (\eta)}^{2}, \sigma_{\ln (\zeta)}^{2}, \sigma_{\ln (\tilde{\varrho})}^{2}$ for means and variances, respectively.

We then have

\footnotetext{
${ }^{6}$ In utility terms this normalization is innocuous because multiplication by $\bar{w}_{t}$ constitutes a monotone transformation of a homothetic utility function.
} 
Proposition 2. Under Assumptions 1-4, a marginal introduction of social security increases social welfare iff

$$
A_{p e, m b r} \equiv \frac{(1+g)}{\bar{R}} \cdot(1+T V)^{\theta}-1>0
$$

where the total variance $T V$ is given as

$$
T V \equiv \operatorname{var}\left(\eta_{i, 1, t} \zeta_{t} \tilde{\varrho}_{t+1}\right)=\underbrace{\sigma_{\eta}^{2}}_{I R}+\underbrace{\sigma_{\zeta}^{2}+\sigma_{\tilde{\varrho}}^{2}+\sigma_{\zeta}^{2} \sigma_{\tilde{\varrho}}^{2}}_{A R}+\underbrace{\sigma_{\eta}^{2}\left(\sigma_{\zeta}^{2}+\sigma_{\tilde{\varrho}}^{2}+\sigma_{\zeta}^{2} \sigma_{\tilde{\varrho}}^{2}\right)}_{L C I=I R \cdot A R} .
$$

Moreover, the consumption equivalent variation associated with the introduction of a social security system with a contribution rate $d \tau$ is

$$
g_{c}= \begin{cases}\left(1+(1-\theta)\left(\frac{1+g}{\bar{R}}(1+T V)^{\theta}-1\right) d \tau\right)^{\frac{1}{1-\theta}}-1 & \text { for } \theta \neq 1 \\ \exp \left(\left(\frac{1+g}{\bar{R}}(1+T V)-1\right) d \tau\right)-1 & \text { for } \theta=1 .\end{cases}
$$

Proof. See Appendix A.

Observe that, according to equation (9), the term $T V$ - abbreviating "total variance"consists of three components, reflecting the effect of idiosyncratic risk in term $I R$, total aggregate risk in term $A R$, and the interaction between idiosyncratic and aggregate risk in term LCI. To see why they appear, notice that in the absence of social security, savings cum interest is given by $a_{i, 2, t+1} R_{t+1}=\bar{w}_{t} \bar{R} \eta_{i, 1, t} \zeta_{t} \tilde{\varrho}_{t+1}$. Hence, from the ex-ante perspective, the product of the three shocks is relevant. This product results from the economic structure of the model, which is completely standard: the aggregate wage is multiplied with the realization of aggregate wage risk, $\zeta_{t}$, and idiosyncratic wage risk, $\eta_{i, 2, t}$, and then savings are multiplied with the realization of aggregate return risk, $\tilde{\varrho}_{t+1}$. The term $T V$ is the variance of the product of these stochastic elements, cf. the product formula of variances derived in Goodman (1960).

For standard random variables, an interaction term involving products of variancessuch as LCI in our context-would be small and is usually ignored. However, we here deal with long horizons so that the single variance terms may well be large. To see this, let us make a rough back of the envelope calculation. Despite the simplicity of our model this gives a rough idea of the magnitudes being involved. Suppose a household works for 40 years, which in the model corresponds to the first period of a household's life. Assume further that each year the household receives a permanent idiosyncratic income 
shock with a $\log$ variance of 1 percent $\left(\sigma_{\ln \eta}^{2}=0.01\right)$, corresponding to standard empirical estimates. Based on this example $L C I$ adds about $(\exp (40 \cdot 0.01)-1) \% \approx 50 \%$ times $A R .^{7}$ Whatever the exact size of $A R$ is, this interaction is clearly a non-negligible increase in overall income risk. Furthermore, observe from equation (10) that the CEV - which, as in Lucas (1978), is a convex function of risk - is equally affected. For the special case of logarithmic utility, the $C E V$ is approximately equal to $\left(\frac{1+g}{\bar{R}}(1+T V)-1\right) d \tau$ so that the 50 percent increase of aggregate risk translates directly into a corresponding increase of the consumption equivalent variation. For risk aversion above one, 50 percent constitutes a lower bound of the contribution of $L C I$ to the $C E V$.

\subsection{Idiosyncratic Risk Late in the Life-Cycle}

Preparing our general equilibrium analysis of the next subsection, we now investigate a version of the model where we consider a subperiod structure for the second period. ${ }^{8}$ The household works in the first subperiod and is retired in the second. To focus the analysis, we rule out idiosyncratic risk in the first period. This implies that we disregard the effect studied in the previous section, i.e., we do not account for the fact that asset positions in the second period inherit components of idiosyncratic and aggregate risk from the first period. Later, when we extend the analysis to general equilibrium, this assumption will be crucial because it allows us to characterize general equilibrium dynamics in closed form. However, idiosyncratic risk hits in the first subperiod of the second period when the household is working. Once we extend the analysis to general equilibrium where consumption and savings decisions also take place in the first period, idiosyncratic risk in the second period will induce (additional) precautionary savings which is an important model element. The following assumption summarizes the simplifications that apply to the current subsection.

Assumption 5. Let $\tilde{\beta}=1, \eta_{i, 1, t}=E \eta_{i, 1, t}=1$ for all $i, t$ and $0 \leq \lambda<1$.

We can now rewrite consumption in the second period of a household's life as

$$
\begin{aligned}
c_{i, 2, t+1}=\bar{w}_{t}\left(\zeta_{t} \bar{R} \varrho_{t+1}\right. & +(1+g) \zeta_{t+1} \eta_{i, 2, t+1} \lambda \\
& \left.+\tau\left((1+g) \zeta_{t+1}\left(1+\lambda\left(1-\eta_{i, 2, t+1}\right)\right)-\zeta_{t} \bar{R} \varrho_{t+1}\right)\right) .
\end{aligned}
$$

\footnotetext{
${ }^{7} \mathrm{By}$ the random walk property of the income process, we have $\sigma_{\ln \eta}=40 \cdot 0.01$. Under $\log$-normality, we have $\sigma_{\eta}^{2}=\exp \left(\sigma_{\ln \eta}\right)-1$.

${ }^{8}$ In the Supplementary Appendix ??, we argue that this is equivalent to a three-period setting. In that three-period setting the results of this subsection go through.
} 
Let's start again by looking at a situation where $\tau=0$ and, without loss of generality, $\bar{w}_{t}=1$. Then, old age consumption becomes

$$
c_{i, 2, t+1}=\underbrace{\zeta_{t} \tilde{\varrho}_{t+1}}_{\equiv \phi} \bar{R}+(1+g) \lambda+(1+g) \lambda(\underbrace{\zeta_{t+1} \eta_{i, 2, t+1}}_{\equiv \psi}-1) .
$$

We now have additive and multiplicative background risk, similar to Franke, Schlesinger, and Stapleton (2011). ${ }^{9}$ A major difference is that they look at a static model where the background risk is additive and multiplicative by construction. By contrast, in our dynamic setting the multiplicative background risk arises endogenously due to the economic structure.

For our purpose, note that both $\psi$ and $\phi$ will have an interaction term, e.g., $\operatorname{var}(\psi)=$ $\operatorname{var}\left(\zeta_{t+1} \eta_{i, 2, t+1}\right)=\sigma_{\zeta}^{2}+\sigma_{\eta}^{2}+\sigma_{\zeta}^{2} \sigma_{\eta}^{2}$. Just like in Proposition 2, the interaction terms will increase the value of a marginal introduction of social security. We can shut down the interaction in both $\psi$ and $\phi$ by assuming $\sigma_{\zeta}=0$, i.e., $\zeta_{t}=E \zeta_{t}=1$ for all $t$. Coincidentally, we then have the more well-known situation with only additive background risk that was originally considered by Gollier and Pratt (1996).

With respect to our thought experiment, we get the following result:

Proposition 3. Under Assumptions 1, 3, and 5, a marginal introduction of social security increases social welfare iff

$$
A_{p e, a m b r}=\mathbb{E}\left[\frac{\frac{1+g}{\bar{R}}(1+\lambda) \frac{\zeta_{t+1}}{\zeta_{t} \varrho_{t+1}}-\frac{1+g}{\bar{R}} \lambda \frac{\zeta_{t+1} \eta_{i, 2, t+1}}{\zeta_{t} \varrho_{t+1}}-1}{\left(\frac{1}{R \zeta_{t} \varrho_{t+1}}\right)^{1-\theta}\left(1+\frac{1+g}{R} \lambda \frac{\zeta_{t+1} \eta_{i, 2, t+1}}{\zeta_{t} \varrho_{t+1}}\right)^{\theta}}\right]>0
$$

Proof. See Appendix A.

Subscript ambr stands in for "additive and multiplicative background risk". In order to simplify the following analysis, we concentrate on the case where $\theta=1$ to the effect that equation (13) becomes

$$
\left.A_{p e, a m b r}\right|_{\theta=1}=\mathbb{E}\left[\frac{\frac{1+g}{\bar{R}}(1+\lambda) \frac{\zeta_{t+1}}{\zeta_{t} \varrho_{t+1}}-\frac{1+g}{\bar{R}} \lambda \frac{\zeta_{t+1} \eta_{i, 2, t+1}}{\zeta_{t} \varrho_{t+1}}-1}{1+\frac{1+g}{R} \lambda \frac{\zeta_{t+1} \eta_{i, 2, t+1}}{\zeta_{t} \varrho_{t+1}}}\right]>0
$$

As we generally assume that $\theta \geq 1$, this constitutes a lower bound for $A_{p e, a m b r}$ because welfare benefits associated with social security increase in $\theta$.

\footnotetext{
${ }^{9}$ The independent, mean-zero shock $\zeta_{t}$ is the multiplicative background risk, because it multiplies the market risk $\tilde{\varrho}_{t+1} \bar{R}$. The independent, mean-zero shock $\zeta_{t+1} \eta_{i, 2, t+1}$ is the additive background risk.
} 
To investigate how interactions of risks affect the term $A_{p e, a m b r}$, we analyze the derivative $\frac{\left.\partial A_{p e, a m b r}\right|_{\theta=1}}{\partial \sigma_{\eta}^{2}}$. If either $\sigma_{\zeta}^{2}$ or $\sigma_{\rho}^{2}$ or both show up positively in this derivative, then idiosyncratic and aggregate risks interact. To derive expressions in closed form we again assume joint log-normality and consider a Taylor-series approximation.

Proposition 4. Consider $\theta=1$. Under Assumptions 1 and 3-5, a second-order Taylor series expansion yields

$$
\frac{\left.\partial A_{p e, a m b r}\right|_{\theta=1}}{\partial \sigma_{\eta}^{2}} \approx \frac{a b^{2}}{(1+b)^{3}}\left(1+\sigma_{\zeta}^{2}\right)^{9}\left(1+\sigma_{\varrho}^{2}\right)^{6}
$$

where $a \equiv(1+\lambda) \frac{1+g}{\bar{R}}, b \equiv \lambda \frac{1+g}{\bar{R}}$.

Proof. See Appendix A.

Hence, we find that in the two period model with a subperiod structure, idiosyncratic and aggregate risks interact, even when there is no idiosyncratic risk in the first period.

\subsection{General Equilibrium}

The previous analyses are restricted to the special case with zero consumption in the first period. In that setting, the value of social security stems from insurance against the risk of income fluctuations. The costs stem from the fact that in a dynamically efficient economy, gross market returns are higher than the implicit return of a PAYG social security system. Two channels are missing in that setting. First, to the extent that social security reduces consumption risk, households need to save less for precautionary motives. This may increase welfare. Second, by crowding out savings, the aggregate capital stock is reduced which suppresses wages and increases returns. This reduces welfare in a dynamically efficient economy. As we will see, the interactions of risks can amplify or mitigate the welfare costs of crowding out.

In order to illustrate these additional channels - and how the interactions of risks affect those-, we consider a setting where consumption decisions are also made in the first period and embed the analysis into a general equilibrium model. For analytical reasons we have to incorporate both steps at once. ${ }^{10}$ We also have to restrict attention

\footnotetext{
${ }^{10}$ In a partial equilibrium model with pension income in the second period - and/or with positive second period labor income in case $\lambda>0$-, the human capital wealth effect inhibits closed form solutions for the saving rate. Our proof of equilibrium dynamics uses the fact that both the interest rate and the wage rate, on which pension payments are based, are functions of the capital stock in general equilibrium.
} 
to $\log _{\text {-utility }}{ }^{11}$ in both periods and assume absence of idiosyncratic shocks in the first period. ${ }^{12}$

Assumption 6. Let $\tilde{\beta} \in\left(0, \frac{1}{2}\right] \Leftrightarrow \beta=\frac{\tilde{\beta}}{1-\tilde{\beta}} \in(0,1] ; u(\cdot)=\ln (\cdot) ; \eta_{i, 1, t}=E \eta_{i, 1, t}=1$ for all $i, t ; 0 \leq \lambda<1$.

\section{General Equilibrium Dynamics}

We begin by characterizing the equilibrium dynamics of the economy.

Proposition 5. Under Assumptions 1 and 6, equilibrium dynamics are given by

$$
\begin{aligned}
k_{t+1} & =\frac{1}{(1+g)(1+\lambda)} s(\tau)(1-\tau)(1-\alpha) \zeta_{t} k_{t}^{\alpha} \\
& =\left(\frac{1}{(1+g)(1+\lambda)} s(\tau)(1-\tau)(1-\alpha)\right)^{\frac{1-\alpha^{\alpha+1}}{1-\alpha}}\left(\prod_{i=0}^{q} \zeta_{t-i}^{\alpha^{i}}\right) k_{t-q}^{\alpha^{1+q}}
\end{aligned}
$$

for some initial capital stock $k_{t-q}$ in period $t-q$. The saving rate, here expressed as a function of $\tau, s(\tau)$, is given by

$$
s(\tau) \equiv \frac{\beta \bar{E}(\tau)}{1+\beta \bar{E}(\tau)} \leq \frac{\beta}{1+\beta} \equiv s(\tau, \lambda=0),
$$

where

$$
\bar{E}(\tau) \equiv \mathbb{E}_{t}\left[\frac{1}{1+\frac{1-\alpha}{\alpha(1+\lambda) \varrho_{t+1}}\left(\lambda \eta_{i, 2, t+1}+\tau\left(1+\lambda\left(1-\eta_{i, 2, t+1}\right)\right)\right)}\right] \leq 1
$$

Proof. See Appendix A.

Notice from (18) that an increase of $\bar{E}$ increases the saving rate. Turning to equation (19) first consider a risk-free situation $\left(\eta_{i, 2, t+1}=\varrho_{t+1}=1\right)$ without a pension system $(\tau=0)$. We then have $\bar{E}=\frac{1}{1+\frac{1-\alpha}{\alpha} \frac{1}{1+\frac{1}{\lambda}}}$. An increase of $\lambda$ leads to higher wage income

This enables us to conveniently rewrite the discounted value of second period labor income (=human capital) so that we can derive closed form solutions for the saving rate and the equilibrium dynamics.

${ }^{11}$ It is crucial that income and substitution effects of changing interest rates offset each other.

${ }^{12}$ In our proof of equilibrium dynamics, we require a homothetic structure. We do not get that with idiosyncratic risk in the first period and a lump-sum pension payment in the second, because the firstperiod wage poor save less than the first-period wage rich. This could be made homothetic by assuming that pension payments do not redistribute across types but then social security no longer insures against idiosyncratic risk. 
in the second period (and a shorter retirement subperiod) which decreases the saving rate by decreasing $\bar{E}$. For $\lambda=0$ we get $\bar{E}=1$.

Next, let's introduce risk while keeping $\tau=0$. Then $\bar{E}=\mathbb{E}_{t}\left[\frac{1}{1+\frac{1-\alpha}{\alpha} \frac{\lambda}{1+\lambda} \frac{\eta_{i, 2, t+1}}{\varrho_{t+1}}}\right]$. Now a mean preserving spread of idiosyncratic shocks, $\eta_{i, 2, t+1}$, increases $\bar{E}$ thereby increasing the saving rate, $s$, as long as $\lambda>0$. This is precautionary savings. By contrast, an increase in the variance of return shocks, $\varrho_{t}$, reduces $\bar{E}$ thereby decreasing the saving rate, $s$. The reason is simply that the asset becomes less attractive, since its risk goes up while the return remains the same.

Finally, let's consider $\tau>0$. Increasing $\tau$ decreases $\bar{E}$ and therefore decreases the saving rate, $s$. This is the crowding-out of private capital formation. Moreover, the larger $\tau$, the smaller the effect of a mean preserving spread of $\eta_{i, 2, t+1}$ on precautionary savings, because of the insurance provided through social security. In the limit case where $\tau=1$, $\eta_{i, 2, t+1}$ has no effect on the saving rate.

\section{Welfare Analysis}

We now turn to a central section of the paper, the welfare analysis in general equilibrium. We look at the same experiment as before, a marginal introduction of a PAYG social security system. In general equilibrium, we can oppose the welfare gains from insurance that we analyzed in the previous sections with the potential welfare losses due to the crowding out of capital.

Proposition 6. Under Assumptions 1 and 6, a marginal introduction of social security increases social welfare in the stationary equilibrium iff

$$
A+B+C>0
$$

where

$$
\begin{aligned}
A & \equiv \beta \mathbb{E}\left[\frac{\frac{(1-\alpha)}{\alpha} \frac{1}{\varrho_{t+1}}-\frac{(1-\alpha) \lambda}{\alpha(1+\lambda)} \frac{\eta_{i, 2, t+1}}{\varrho_{t+1}}-1}{1+\frac{(1-\alpha) \lambda}{\alpha(1+\lambda)} \frac{\eta_{i, 2, t+1}}{\varrho t+1}}\right]-1 \\
B & \equiv \frac{\partial s(\tau)}{\partial \tau}\left(\left.\beta \frac{1}{s(\tau=0)} \bar{E}\right|_{\tau=0}-\frac{1}{1-s(\tau=0)}\right)=0 \\
C & \equiv-\left(\alpha(1+\beta)-\left.\beta(1-\alpha) \bar{E}\right|_{\tau=0}\right) \frac{1}{1-\alpha} \underbrace{\left(1-\left.\epsilon_{s, \tau}\right|_{\tau=0}\right)}_{>0}
\end{aligned}
$$


where

$$
\left.\epsilon_{s, \tau}\right|_{\tau=0} \equiv \frac{\partial s / s}{\partial \tau}=-\frac{\left.\overline{\bar{E}}\right|_{\tau=0}}{\left.\left(1+\left.\beta \bar{E}\right|_{\tau=0}\right) \bar{E}\right|_{\tau=0}}<0
$$

is the semi-elasticity of the saving rate $s$ with respect to the contribution rate evaluated at $\tau=0$ and terms $\bar{E}$ and $\overline{\bar{E}}$ are given by

$$
\begin{aligned}
& \left.\bar{E}\right|_{\tau=0} \equiv \mathbb{E}\left[\frac{1}{1+\frac{(1-\alpha) \lambda}{\alpha(1+\lambda)} \frac{\eta_{i, 2, t+1}}{\varrho_{t+1}}}\right]>0 \\
& \left.\overline{\bar{E}}\right|_{\tau=0} \equiv-\left.\frac{\partial \bar{E}}{\partial \tau}\right|_{\tau=0}=\mathbb{E}\left[\frac{\frac{1-\alpha}{\alpha(1+\lambda)} \frac{1+\lambda\left(1-\eta_{i, 2, t+1}\right)}{\varrho_{t+1}}}{\left(1+\frac{(1-\alpha) \lambda}{\alpha(1+\lambda)} \frac{\eta_{i, 2, t+1}}{\varrho_{t+1}}\right)^{2}}\right]>0 .
\end{aligned}
$$

Proof. See Appendix A.

In the above, term $A$ reflects the rate of return condition of social security and thus is the general equilibrium analogue to the partial equilibrium term $A_{p e, a m b r}$ from equation (14). There are two differences between the two. First, in general equilibrium, the interest rate is determined endogenously. Second, in general equilibrium the aggregate productivity shocks, $\zeta_{t}$ and $\zeta_{t+1}$, drop out. Intuitively, this happens because that shock affects all sources of income, namely wages, returns, and social security pensions. As shown in the Supplementary Appendix ??, the aggregate productivity shocks can be separated from the contribution rate, $\tau$, and all endogenous variables, so that it drops out in the analysis of marginal effects.

Similar to the analysis of Subsection 3.2 term $A$ depicts the trade off between the insurance gains due to social security and the welfare losses due to the fact that the implicit return of social security is less than the expected return on savings in a dynamically efficient economy. This will become more clear below. We make two more observations regarding term $A$. First, term $A$ does not capture any behavioral responses to the policy reform. Second, it increases in $\beta$, because households care more about consumption risk in the second period when $\beta$ is higher.

Term $B$ represents the utility effects of the precautionary savings reaction. There are two opposing forces which exactly offset each other in utility terms. On the one hand, households need less precautionary savings, because they have more insurance, and as a consequence can consume more in the first period of life. On the other hand, the reduction of precautionary savings leads to a reduction of aggregate capital. As a 
direct consequence, wages and returns change, and this reduces utility in such a way that the net effect is zero. We give more details on this somewhat surprising finding in the Supplementary Appendix ??.

Term $C$ represents the welfare effects due to crowding out of capital formation. It thus captures the response of households to a reduction in their income. It increases in $\beta$, which means that the more households value future consumption, the less they will reduce their savings in response to lower income. Whether term $C$ is positive or negative depends on whether the economy is dynamically efficient. This is formalized in the next proposition.

Lemma 1. Consider a deterministic economy with $\lambda=0$. This economy is dynamically efficient in the sense of Cass (1972) iff

$$
s(\tau=0, \lambda=0)=\frac{\beta}{1+\beta}<\frac{\alpha}{1-\alpha} .
$$

Proposition 7. If condition (23) holds in the deterministic economy with $\lambda=0$, then term $C<0$ in the corresponding stochastic economy with $0 \leq \lambda<1$.

Proof. See Appendix A. The lemma is proved as part of the proposition.

The proposition connects the classic notion of dynamic efficiency due to Cass (1972) to the welfare effect of crowding out in our stochastic economy. If the deterministic version of the economy is dynamically efficient, then the crowding out of capital leads to a welfare loss, i.e., term $C<0$.

To sum up the discussion on the three terms, in a dynamically efficient economy, the introduction of social security will increase welfare due to insurance, reflected by term $A$, but it will reduce welfare due to the crowding out of capital, reflected by term $C$. In the following, we discuss how the interactions between risks affect the two terms. To this end, we analyze the derivatives $\frac{\partial A}{\partial \sigma_{\eta}^{2}}$ and $\frac{\partial C}{\partial \sigma_{\eta}^{2}}$. If $\sigma_{\rho}^{2}$ shows up positively in the respective derivatives, then idiosyncratic and aggregate risks interact, just as in our previous partial equilibrium analysis of Subsection 3.2. In order to derive analytical expressions we modify Assumption 4 and again consider a Taylor series expansions of the random variables around their respective means. ${ }^{13}$

Assumption 7. Joint log-normality: $\eta_{i, 2, t}, \varrho_{t+1}$ are jointly distributed as log-normal with parameters $\mu_{\ln \eta}, \mu_{\ln \varrho}, \sigma_{\ln (\eta)}^{2}, \sigma_{\ln (\varrho)}^{2}$ for means and variances, respectively.

\footnotetext{
${ }^{13}$ In the Supplementary Appendix ??, we examine the special case of $\lambda=0$, which yields concise equations without the need for an additional assumption.
} 
Proposition 8. Under Assumptions 1, 6, and 7, a second-order Taylor series expansion yields

$$
\begin{aligned}
& \frac{\partial A}{\partial \sigma_{\eta}^{2}} \approx \beta \frac{a^{2} b\left(1+\sigma_{\varrho}^{2}\right)^{6}}{(1+a)^{3}}>0 \\
& \frac{\partial C}{\partial \sigma_{\eta}^{2}} \approx \underbrace{\left(\alpha(1+\beta)-\left.\beta(1-\alpha) \bar{E}\right|_{\tau=0}\right)}_{>0} \frac{1}{1-\alpha} \underbrace{\frac{\left.\partial \epsilon_{s, \tau}\right|_{\tau=0}}{\partial \sigma_{\eta}^{2}}}_{<0}+\beta \underbrace{\left(1-\left.\epsilon_{s, \tau}\right|_{\tau=0}\right)}_{>0} \underbrace{\frac{\left.\partial \bar{E}\right|_{\tau=0}}{\partial \sigma_{\eta}^{2}}}_{>0} \gtrless 0
\end{aligned}
$$

where

$$
\begin{aligned}
a & \equiv \frac{(1-\alpha) \lambda}{\alpha(1+\lambda)}, b \equiv \frac{(1-\alpha)}{\alpha} \\
\frac{\left.\partial \bar{E}\right|_{\tau=0}}{\partial \sigma_{\eta}^{2}} & =\frac{a^{2}\left(\sigma_{\varrho}^{2}+1\right)^{3}}{(1+a)^{3}}>0 \\
\frac{\left.\partial \epsilon_{s, \tau}\right|_{\tau=0}}{\partial \sigma_{\eta}^{2}} & \approx-\beta \frac{(1-s)^{2}}{s} . \\
& \cdot\left(\left(\sigma_{\varrho}^{2}+1\right)^{3} \frac{3 a^{2} b\left(\sigma_{\varrho}^{2}+1\right)^{3}-a^{3}+2 a^{2}}{(a+1)^{4}}+\frac{a^{2}\left(\sigma_{\varrho}^{2}+1\right)^{3}}{(1+a)^{3}}\right)<0
\end{aligned}
$$

Proof. See Appendix A.

To interpret the previous proposition, first observe that our findings with regard to term $A$ are analogous to our partial equilibrium results from Subsection 3.2: Welfare benefits from introducing social security interact positively with aggregate risk. This is reflected by the variance $\sigma_{\varrho}^{2}$ in the partial derivative $\frac{\partial A}{\partial \sigma_{\eta}^{2}}$.

Second, notice that the effect of idiosyncratic risk on the welfare losses from crowding out is ambiguous. The ambiguity comes from the fact that (24) is positive whereas (25) is negative. Both terms are positively interacting with aggregate risk. This can be seen by the presence of term $\sigma_{\varrho}^{2}$ in the respective expressions. Term (25) is the derivative of the semi-elasticity of the saving rate with respect to idiosyncratic risk. It is negative because the reduction of savings in response to an introduction of the pension system is stronger when both risks are present. The underlying reason is that social security can partially insure both risks, which means that precautionary savings decrease more strongly than if only one risk was insured. The interaction terms reinforce this channel. The opposing force is given in equation (24) which displays the partial derivative of $\left.\bar{E}\right|_{\tau=0}$ 
with respect to $\sigma_{\eta}^{2}$, which has a positive sign. Recall from equation (18) that an increase of $\left.\bar{E}\right|_{\tau=0}$ is equivalent to an increase of the saving rate. Equation (24) hence captures a level effect, i.e., that savings itself is higher when both risks are present. Since this equation increases in $\sigma_{\varrho}^{2}$, the risk interactions mitigate this channel and thereby mitigate the losses from crowding out.

\section{Numerical Illustration}

This section presents a numerical illustration of the results of the previous section. Specifically, it illustrates the interactions of risks as documented in Proposition 2 for the partial and in Proposition 6 for the general equilibrium. The aim is to gain qualitative insights, not to perform a rigorous quantitative exercise.

We focus on results for the terms $A_{p e, m b r}, A$, and $C$. To underscore that our analysis is qualitative in nature, we don't report the associated (contributions to) consumption equivalent variations because these have a stronger quantitative connotation. However, recall from Proposition 2 that our findings on these terms translate one for one into corresponding findings for consumption equivalent variations.

We parameterize the model such that each period covers $J=40$ actual years. We set $\alpha=0.3$ and $\beta=0.99^{J}$. With these parameters, the sufficient condition of dynamic efficiency in Proposition 7 is satisfied. We set the log variance of innovations of the idiosyncratic income process to an annual value of 0.01 , corresponding to conventional estimates. Given the periodicity of $J=40$ years, this means that $\sigma_{\eta}^{2}=\exp (40 \cdot 0.01)-$ $1 \approx 0.5$. The variance of total aggregate risk $(A R)$ is assumed to be $\in[0,1]$ with details described below. Furthermore, we set $\lambda=0.1$ which assigns a relatively big role to social security - i.e., the pension period with weight $1-\lambda$ is relatively longand a small role to idiosyncratic risk - i.e., the working phase with weight $\lambda$ is relatively short. Furthermore, $1+g=(1+0.015)^{J}$, which is a standard value for the long run real productivity growth rate. For the partial equilibrium the coefficient of relative risk aversion can take two values, $\theta \in\{1,3\}$, whereas for the general equilibrium we keep in line with the proposition and let $\theta=1$.

\subsection{Partial Equilibrium}

The partial equilibrium version of the model was presented in Subsection 3.2. To keep the results comparable to the general equilibrium, we set the gross interest rate $\bar{R}$ to a 
value that would result from a general equilibrium where all realizations of shocks equal their respective means in a $\lambda=0$ economy. Details are relegated to the Supplementary Appendix ??, see in particular Definition ?? and equation (??). This yields $\bar{R}=(1+$ g) $\frac{\alpha}{1-\alpha} \frac{1+\beta}{\beta}=1.9396$ for the parameterization explained above. For this value, $\frac{\bar{R}}{1+g}=$ $1.0692>1$, i.e., the capital market return exceeds the implicit return of social security.

Figure 1 displays the term $A_{p e, m b r}$ of Proposition 2 for $\theta=1$ in Panel (a) and for $\theta=2$ in Panel (b) as a function of the standard deviation of aggregate risk, $\sqrt{A R}$. Each point in the graph has $\sigma_{\zeta}=\sigma_{\varrho}{ }^{14}$

The solid black line in the figure is for the case with only aggregate risk, $A_{p e, m b r}(A R)$, whereas the red dash-dotted line is for both risks, $A_{p e, m b r}(A R, I R)$. The blue dashed line shows $A_{p e, m b r}(A R+I R)$, which can be interpreted as a scenario with a single risk that has the size of the two separate risks. Crucially, in this scenario $L C I=0$. We can get an analytical expression for $A_{p e, m b r}(A R+I R)$ from equation 8: for $\theta=1$ we have $A_{p e, m b r}(A R+I R)=\frac{1+g}{\bar{R}}+\frac{1+g}{\bar{R}} A R+\frac{1+g}{\bar{R}} I R-1$. This is displayed in Panel (a). Since we hold $I R$ constant, the shift by $\frac{1+g}{\bar{R}} I R$ remains unchanged as $\sqrt{A R}$ is increased, to that the curves move closer together. By contrast, for $\theta=3$ in Panel (b), we get $A_{p e, m b r}(A R+I R)=\frac{1+g}{\bar{R}}(1+A R+I R)^{3}-1$ which means that the degree of convexity increases. Importantly, observe that in both graphs the gap between the red dash-dotted line, $A_{p e, m b r}(A R, I R)$, and the blue dashed line, $A_{p e, m b r}(A R+I R)$, is increasing. This amplification is caused by the interaction of risks through $L C I$. The amplification increases in risk aversion, cf. equation (8).

Finally, notice that $A_{p e, m b r}(A R)$ turns from negative-because $\frac{\bar{R}}{1+g}>1$ - to positive for sufficiently high aggregate risk. $A_{p e, m b r}(A R, I R)$ is positive throughout because the insurance against idiosyncratic risk dominates the welfare losses in this dynamically efficient economy already for $A R=0$.

\footnotetext{
${ }^{14}$ We therefore have $A R=\sigma_{\zeta}^{2}+\sigma_{\varrho}^{2}+\sigma_{\zeta}^{2} \sigma_{\varrho}^{2}=2 \sigma_{\zeta}^{2}+\sigma_{\zeta}^{4}$, cf. Proposition 2. This gives $\sigma_{\zeta}^{2}=\sigma_{\varrho}^{2}=$ $-1+\sqrt{1+A R}$. As we assume that $A R \in[0,1]$ this implies that that $\sigma_{\zeta}^{2}$ and $\sigma_{\varrho}^{2}$ are $\in[0,0.41]$.
} 
Figure 1: Welfare Effects in Partial Equilibrium
(a) $\theta=1$
(b) $\theta=3$
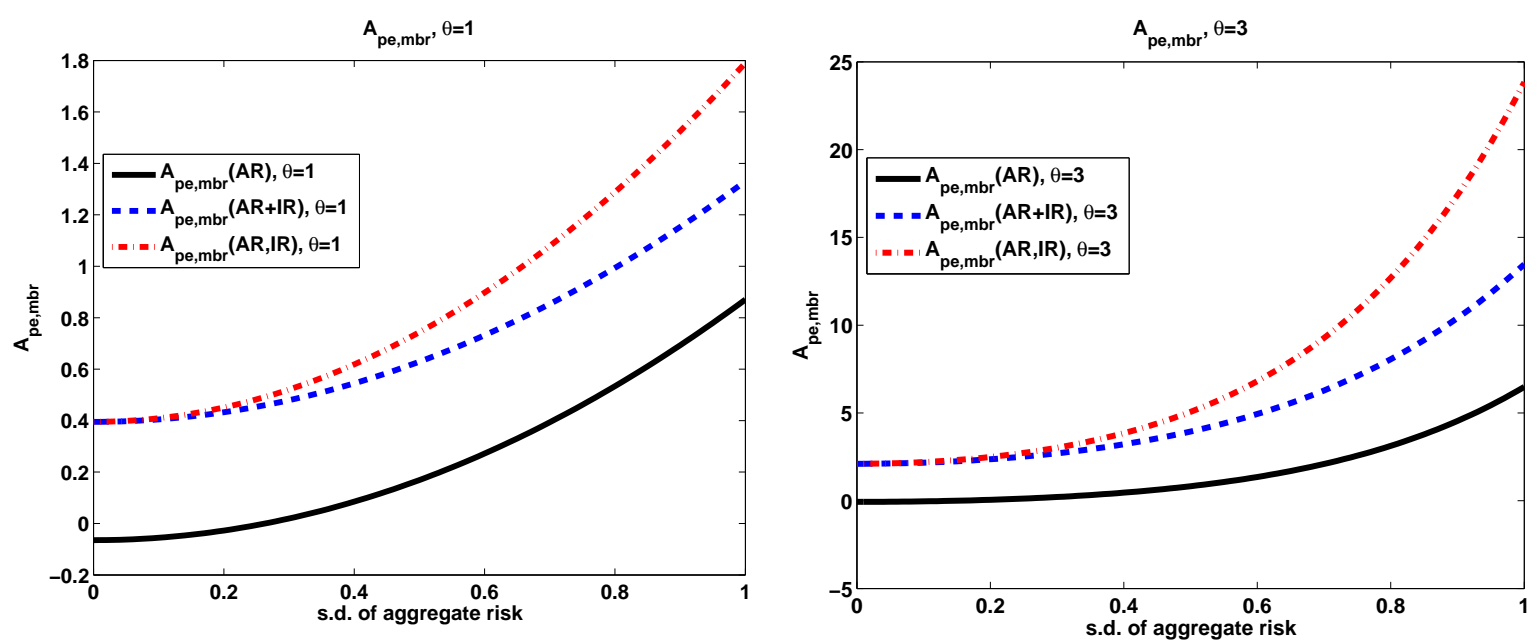

Notes: $A_{p e, m b r}$ is defined in equation (7). We set the variance of aggregate return shocks $\sigma_{\varrho}=\sigma_{\zeta}$. Aggregate risk, $A R$ is then $A R=\sigma_{\zeta}^{2}+\sigma_{\zeta}^{2}+\sigma_{\zeta}^{4}$, cf. Proposition 2. $A_{p e, m b r}(A R)$ is for an economy with only aggregate risk, $A_{p e, m b r}(A R+I R)$ is for an economy with a single risk that has the size of both risks, and $A_{p e, m b r}(A R, I R)$ is the economy with two separate risks. $\theta$ is the coefficient of relative risk aversion. 


\subsection{General Equilibrium: Comparative Statics}

We now turn to the analysis in general equilibrium where we compare two long-run equilibria like in Proposition 6. Recall that in general equilibrium, we have $\lambda \geq 0$ and we here set $\lambda=0.1$ (see above).

We here present numerical computations of the expressions in Proposition 6, not using the approximations of Proposition 8. To this end we compute the expected values of all non-linear expressions by Gaussian Quadrature methods. We evaluate the integrals using $n_{p}=5$ nodes. We know from Proposition 6 that no aggregate productivity shocks are present. Therefore, in contrast to the previous subsection, aggregate risk is simply given by $A R=\sigma_{\varrho}^{2}$. To keep $A R \in[0,1]$ as previously we accordingly now set $\sigma_{\varrho}^{2} \in[0,1] .{ }^{15}$

Figure 2 displays the terms $A$ and $C$, as well as the total effect $A+C$ as a function of $\sqrt{A R}=\sigma_{\varrho}$. With regard to term $A$, shown in panel (a) of the figure, we see a similar qualitative pattern as in our partial equilibrium model. Concretely, the solid black line $A(A R)$ corresponds to the solid black line $A_{p e, m b r}(A R)$ in panel (a) of Figure 1. Both lines are strictly increasing and convex, start below zero, and become positive as we increase aggregate risk. The two lines are not the same because $A_{p e, m b r}$ corresponds to $\lambda=0$, whereas $A$ is plotted for $\lambda=0.1$, cf. Footnote 15. Also the red dash-dotted lines, $A(A R, I R)$ and $A_{p e, m b r}(A R, I R)$, which represent economies with both risks, behave the same. But the crucial feature is that we see again that the gap between the two lines increases, which is due to the presence of interactions between risks, since we keep idiosyncratic risk constant.

With regard to term $C$, the presence of idiosyncratic risk turns out to reduce the welfare costs from crowding out, i.e., the red dash-dotted line is above the black solid line. However, this is ambiguous. As aggregate risk increases, the gap becomes smaller, which means that the interactions increase the welfare costs from crowding out. This corresponds to our finding in Proposition 8, where we discussed the two opposing forces and concluded that the interactions could increase or decrease the welfare losses in $C$.

Finally, we see that the total effect, $A+C$, displayed in Panel (c) is increasing in aggregate risk. This is so because the marginal welfare benefits from insurance (term $A$ ) dominate the marginal welfare losses from crowing out (term $C$ ) for every unit of additional aggregate risk. More importantly, the gap between the two lines in Panel (c) increases, which means that the interactions in term $A$ dominate those in term $C$. While

\footnotetext{
${ }^{15}$ Also notice that in the $\lambda=0$ economy we have $A_{p e, m b r}=\frac{A}{1+\beta}$, with details relegated to Supplementary Appendix ??. The following results therefore show terms $A$ and $C$ times $\frac{1}{1+\beta}$.
} 
we are looking only at a specific calibration, this is still an important finding (and, in fact, a very robust finding across different calibrations which we do not show for sake of brevity). Interactions between the risks seem to amplify the welfare gains more strongly than the welfare losses. The reason are the two opposing forces in term $C$ shown and discussed in Proposition 8. Because of them, the effect of the interactions on the welfare costs is mild, and the effect on the welfare gains dominates.

Figure 2: Welfare Effects in General Equilibrium

(a) Term A

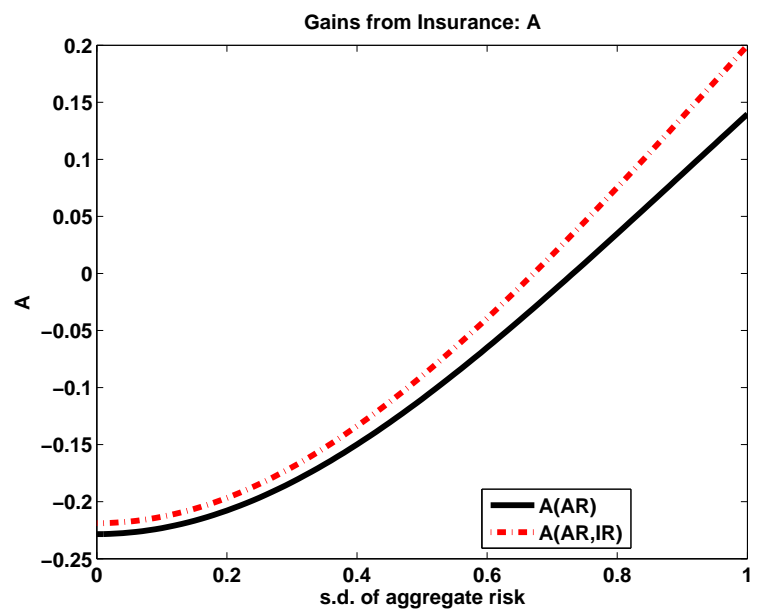

(b) Term C

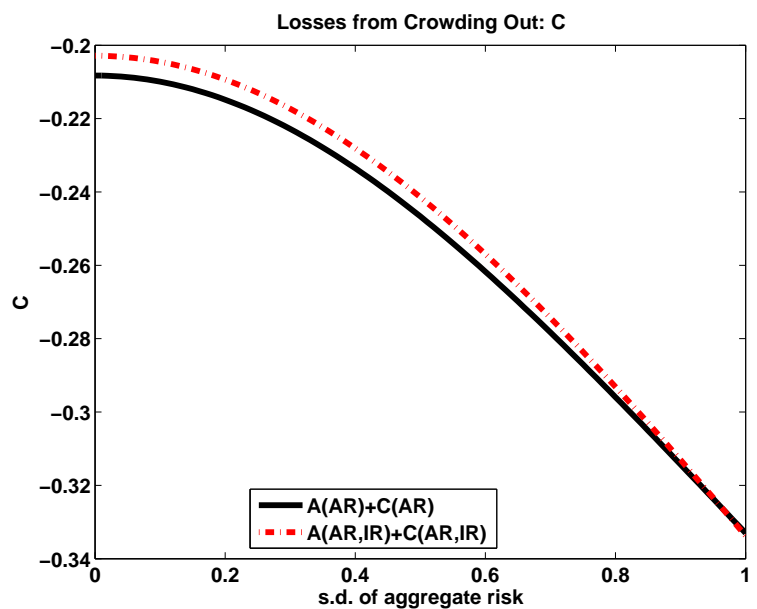

(c) Total Effect

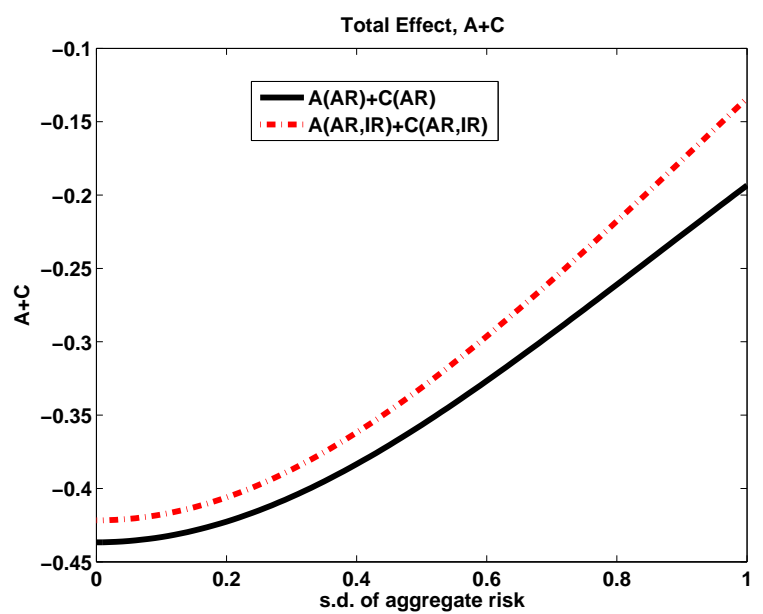

Notes: $A$ and $C$ are defined in proposition (6). Aggregate risk is $A R=\sigma_{o}^{2}$, because $\sigma_{\zeta}$ doesn't enter in (6). $A(A R), C(A R)$ are for an economy with only aggregate risk, and $A(A R, I R), C(A R, I R)$ are for the economy with two separate risks. 


\section{Conclusion}

This paper develops an analytically tractable model with two overlapping generations where households are subject to aggregate and idiosyncratic risk. We use this model to study the welfare consequences of introducing a marginal pay-as-you-go social security system. We highlight important interactions between aggregate and idiosyncratic risks which are present although these risks are orthogonal by construction. It is the standard life-cycle structure of the economy that causes these interactions. They increase the variance of retirement consumption and therefore increase the insurance value of social security. We first demonstrate this insurance channel in a partial equilibrium. There, we show that the two risks interact in multiple ways which all drive up the value of social security. Then, in general equilibrium, we oppose this same insurance channel with the welfare loss from the crowding out of capital which arises due to distortionary taxation. The crucial finding here is that when two risks are present, the crowding out is the result of two opposing forces. As a consequence, the interactions act less strong on the welfare costs of crowding out than they do on the welfare gains from insurance. This is confirmed in our numerical illustrations, where the net effect of the interactions on welfare in general equilibrium is positive.

The model makes contact with the literature on background risk and extends it to a dynamic setting. First, by construction we have additive background risk like in Gollier and Pratt (1996). However, due to our dynamic setting, we can show that the additive background risk increases because the independent idiosyncratic and aggregate productivity risks interact. Second, we also show that the model has multiplicative background risk like in Franke, Schlesinger, and Stapleton (2006). However, in that paper it is present by direct construction, whereas in our model it arises due to the economic structure. Also for this multiplicative background risk, we show that there is an interaction term.

The findings in this paper suggest that welfare effects stemming from these interactions of risks may in fact be quite be large. When aggregate risk in the economy is sufficiently strong, this may lead to welfare gains from the introduction of social security also in the long-run despite losses from crowding out of capital formation. Whether this is the case must be investigated in a more realistic quantitative model which is suitably calibrated. This is done in our companion paper, Harenberg and Ludwig (2014). There we document that indeed the interactions of risks overturn conventional findings on the welfare effects of social security and we conclude that the introduction of a minimum flat pension is welfare improving once all household risks are appropriately taken into account. 


\section{References}

Aaron, H. J. (1966). The Social Insurance Paradox. Canadian Journal of Economics and Political Science 32, 371-374.

Auerbach, A. J. and K. Hassett (2007). Optimal Long-Run Fiscal Policy: Constraints, Preferences and the Resolution of Uncertainty. Journal of Economic Dynamics and Control 31, 1451-1472.

Ball, L. and N. G. Mankiw (2007). Intergenerational risk sharing in the spirit of Arrow, Debreu, and Rawls, with applications to social security design. Journal of Political Economy 115(4), 523-547.

Bohn, H. (2001). Social Security and Demographic Uncertainty: The Risk Sharing Properties of Alternative Policies. In J. Campbell and M. F. Feldstein (Eds.), Risk Aspects of Investment Based Social Security Reform, pp. 203-241. University of Chicago Press.

Bohn, H. (2009). Intergenerational Risk Sharing and Fiscal Policy. Journal of Monetary Economics 56, 805-816.

Caliendo, F. N., L. N. Guo, and R. Hosseini (2013). Social Security is NOT a Substitute for Annuities.

Cass, D. (1972). On Capital Overaccumulation in the Aggregative, Neoclassical Model of Economic Growth: A Complete Characterization. Journal of Economic Theory $4(2), 200-223$.

Conesa, J. C. and D. Krüger (1999). Social Security Reform with Heterogenous Agents. Review of Economic Dynamics 2, 757-795.

De Santis, M. (2007). Individual Consumption Risk and the Welfare Cost of Business Cycles. Amercian Economic Review 97(4), 1488-1506.

Diamond, P. A. (1965). National Debt in a Neoclassical Growth Model. American Economic Review 55, 1126-1150.

Diamond, P. A. (1977). A framework for social security analysis. Journal of Public Economics 8(3), 275-298.

Franke, G., H. Schlesinger, and R. C. Stapleton (2006). Multiplicative Background Risk. Management Science 52, 146-153. 
Franke, G., H. Schlesinger, and R. C. Stapleton (2011, July). Risk taking with additive and multiplicative background risks. Journal of Economic Theory 146(4), 15471568.

Gollier, C. and J. W. Pratt (1996). Risk Vulnerability and the Tempering Effect of Background Risk. Econometrica 64, 1109-1123.

Goodman, L. A. (1960). On the Exact Variance of Products. Journal of the American Statistical Association 55(292), 708-713.

Gordon, R. H. and H. R. Varian (1988). Intergenerational Risk Sharing. Journal of Public Economics 37(2), 185-202.

Harenberg, D. and A. Ludwig (2014). Social Security and the Interactions Between Aggregate and Idiosyncratic Risk.

Harenberg, D., A. Ludwig, and J. Maus (2013). The Welfare Effects of Social Security in a Model with Aggregate and Idiosyncratic Risk.

Hasanhodzic, J. and L. J. Kotlikoff (2013). Generational Risk: Is It a Big Deal?: Simulating an 80-Period OLG Model with Aggregate Shocks.

Huffman, G. W. (1987). A Dynamic Equilibrium Model of Asset Prices and Transaction Volume. Journal of Political Economy 95(1), 138-159.

Huggett, M. and G. Ventura (1999). On the Distributional Effects of Social Security Reform. Review of Economic Dynamics 2, 498-531.

Imrohoroglu, A., S. Imrohoroglu, and D. H. Joines (1995). A Life Cycle Analysis of Social Securtiy. Economic Theory 6, 83-114.

Imrohoroglu, A., S. Imrohoroglu, and D. H. Joines (1998). The Effect of Tax-Favored Retirement Accounts on Capital Accumulation. American Economic Review 88(4), $749-768$.

Krebs, T. (2007). Job Displacement Risk and the Cost of Business Cycles. The American Economic Review 97(3), 664-686.

Krüger, D. and F. Kübler (2006). Pareto Improving Social Security Reform When Financial Markets are Incomplete!? American Economic Review 96(3), 737-755.

Lucas, R. E. (1978). Asset Prices in an Exchange Economy. Econometrica 46(6), 14261445.

Ludwig, A. and M. Reiter (2010). Sharing Demographic Risk - Who is Afraid of the Baby Bust? American Economic Journal: Economic Policy 2(4), 83-118. 
Ludwig, A. and E. Vogel (2009). Mortality, Fertility, Education and Capital Accumulation in a Simple $\{$ OLG $\}$ Economy. Journal of Population Economics.

Matsen, E. and O. Thogersen (2004). Designing Social Security - a Portfolio Choice Approach. European Economic Review 48, 883-904.

Merton, R. C. (1983). On the Role of Social Security as a Means for Efficient Risk Sharing in an Economy Where Human Capital Is Not Tradable. In Z. Bodie and J. B. Shoven (Eds.), Financial aspects of the United States pension system, pp. 325-58. Chicago: University of Chicago Press.

Olovsson, C. (2010). Quantifying the Risk-Sharing Welfare Gains of Social Security. Journal of Monetary Economics 57, 364-375.

Shiller, R. J. (1999). Social Security and Institutions for Intergenerational, Intragenerational, and International Risk-Sharing. Carnegie-Rochester Conference Series on Public Policy 50(99), 165-204.

Storesletten, K. C., C. Telmer, and A. Yaron (1999). The Risk-Sharing Implications of Alternative Social Security Arrangements. Carnegie-Rochester Conference Series on Public Policy 50, 213-259. 


\section{A Appendix: Proofs}

Proof of proposition 1. Maximize

$$
\mathbb{E} u\left(c_{i, 2, t+1}\right)=\frac{1}{1-\theta} \mathbb{E}\left(\bar{w}_{t}\left(\bar{R} \eta_{i, 1, t} \zeta_{t} \tilde{\varrho}_{t+1}+\tau\left((1+g) \zeta_{t+1}-\bar{R} \eta_{i, 1, t} \zeta_{t} \tilde{\varrho}_{t+1}\right)\right)\right)^{1-\theta} .
$$

This is equivalent to maximizing

$$
\frac{1}{1-\theta} \max \mathbb{E} R_{p, t, t+1}^{1-\theta}
$$

where $R_{p, t, t+1} \equiv \eta_{i, 1, t} \zeta_{t} \bar{R} \tilde{\varrho}_{t+1}+\tau\left((1+g) \zeta_{t+1}-\bar{R} \eta_{i, 1, t} \zeta_{t} \tilde{\varrho}_{t+1}\right)$ is a consumption (or portfolio) return. Increasing ex-ante utility for a marginal introduction of social security requires the first-order condition w.r.t. $\tau$, evaluated at $\tau=0$, to exceed zero, hence:

$$
\left.\mathbb{E}\left[R_{p, t, t+1}^{-\theta} \frac{\partial R_{p, t, t+1}}{\partial \tau}\right]\right|_{\tau=0}>0
$$

We have $\left.R_{p, t, t+1}^{-\theta}\right|_{\tau=0}=\left(\eta_{i, 1, t} \zeta_{t} \bar{R} \varrho_{t+1}\right)^{-\theta}$ and $\left.\frac{\partial R_{p, t, t+1}}{\partial \tau}\right|_{\tau=0}=(1+g) \zeta_{t+1}-\eta_{i, 1, t} \zeta_{t} \bar{R} \varrho_{t+1}$. Equation (26) therefore rewrites as

$$
(1+g) \mathbb{E}\left[\left(\eta_{i, 1, t} \zeta_{t} \varrho_{t+1}\right)^{-\theta}\right]>\bar{R} \mathbb{E}\left[\left(\eta_{i, 1, t} \zeta_{t} \varrho_{t+1}\right)^{1-\theta}\right]
$$

Rewriting the above and imposing Assumption 1 we get equation (7).

Proof of proposition 2. Define $Z_{1} \equiv\left(\eta_{i, 1, t} \zeta_{t} \tilde{\varrho}_{t+1}\right)^{-\theta}$ and $Z_{2} \equiv\left(\eta_{i, 1, t} \zeta_{t} \tilde{\varrho}_{t+1}\right)^{1-\theta}$. By lognormality we have that $\mathbb{E} Z_{i}=\exp \left(E \ln Z_{i}+\frac{1}{2} \sigma_{\ln Z_{i}}^{2}\right), i=1,2$. Observe that $\mathbb{E} \ln Z_{1}=$ $-\theta\left(\mathbb{E} \ln \eta_{i, 1, t}+\mathbb{E} \ln \varrho+\mathbb{E} \ln \zeta\right)$ and $\sigma_{\ln Z_{1}}^{2}=\theta^{2}\left(\sigma_{\ln \eta}^{2}+\sigma_{\ln \varrho}^{2}+\sigma_{\ln \zeta}^{2}\right)$. Therefore:

$$
\mathbb{E}\left[Z_{1}\right]=\exp \left(\frac{1}{2} \theta(1+\theta)\left(\sigma_{\ln \eta}^{2}+\sigma_{\ln \tilde{\varrho}}^{2}+\sigma_{\ln \zeta}^{2}\right)\right)
$$

where we make use of Assumption 1b. Next, observe that log-normality implies that $\sigma_{\eta}^{2}=$ $\left(\exp \left(\sigma_{\ln \eta}^{2}\right)-1\right)$, again using Assumption 1b. Hence $\sigma_{\ln \eta}^{2}=\ln \left(1+\sigma_{\eta}^{2}\right)$ with corresponding expressions for $\sigma_{\ln \zeta}^{2}$ and $\sigma_{\ln \tilde{\varrho}}^{2}$. Therefore:

$$
\exp \left(\frac{1}{2} \theta(1+\theta)\left(\sigma_{\ln \eta}^{2}+\sigma_{\ln \varrho}^{2}+\sigma_{\ln \zeta}^{2}\right)\right)=\left(\left(1+\sigma_{\eta}^{2}\right)\left(1+\sigma_{\zeta}^{2}\right)\left(1+\sigma_{\tilde{\varrho}}^{2}\right)\right)^{\frac{1}{2} \theta(1+\theta)}
$$

We consequently have $\mathbb{E}\left[Z_{1}\right]=\left(\left(1+\sigma_{\eta}^{2}\right)\left(1+\sigma_{\zeta}^{2}\right)\left(1+\sigma_{\tilde{\varrho}}^{2}\right)\right)^{\frac{1}{2} \theta(1+\theta)}$. 
As to $\mathbb{E}\left[Z_{2}\right]$ observe that $\mathbb{E} \ln Z_{2}=(1-\theta)\left(\mathbb{E} \ln \eta_{i, 1, t}+\mathbb{E} \ln \zeta+\mathbb{E} \ln \varrho\right)$ and $\sigma_{\ln Z_{2}}^{2}=$ $(1-\theta)^{2}\left(\sigma_{\ln \eta}^{2}+\sigma_{\ln \zeta}^{2}+\sigma_{\ln \tilde{\varrho}}^{2}\right)$. Therefore $\mathbb{E}\left[Z_{2}\right]=\left(\left(1+\sigma_{\eta}^{2}\right)\left(1+\sigma_{\zeta}^{2}\right)\left(1+\sigma_{\tilde{\varrho}}^{2}\right)\right)^{\frac{1}{2} \theta(\theta-1)}$ and hence $\frac{\mathbb{E}\left[Z_{1}\right]}{\mathbb{E}\left[Z_{2}\right]}=\left(\left(1+\sigma_{\eta}^{2}\right)\left(1+\sigma_{\zeta}^{2}\right)\left(1+\sigma_{\tilde{\varrho}}^{2}\right)\right)^{\theta}$.

To evaluate the CEV between two scenarios we compare $\mathbb{E} u\left(c_{i, 2, t+1_{\tau}>0}\right)$ with $\mathbb{E} u\left(c_{i, 2, t+1_{\tau=0}}\right)$ using that $\mathbb{E} u\left(c_{i, 2, t+1_{\tau}>0}\right) \approx \mathbb{E} u\left(c_{i, 2, t+1_{\tau=0}}\right)+\frac{\partial \mathbb{E} u\left(c_{i, 2, t+1_{\tau=0}}\right)}{\partial \tau} d \tau$. We evaluate this expression at $\tau=0$. We start by looking at general CRRA utility, proceeding with the log case.

1. Case $\theta \neq 1$. We have that, evaluated at $\tau=0$,

$$
\frac{\partial \mathbb{E} u\left(c_{i, 2, t+1_{\tau=0}}\right)}{\partial \tau}=\bar{w}_{t}^{1-\theta} \bar{R}^{1-\theta}\left(\frac{1+g}{\bar{R}} \mathbb{E} Z_{1}-\mathbb{E} Z_{2}\right)
$$

where $Z_{1}$ and $Z_{2}$ are defined in our proof of Proposition 2. We also have that $\mathbb{E} u\left(c_{i, 2, t+1}{ }_{\tau=0}\right)=\frac{1}{1-\theta} \bar{w}_{t}^{1-\theta} \bar{R}^{1-\theta} \mathbb{E} Z_{2}$. Therefore:

$$
\mathbb{E} u\left(c_{i, 2, t+1}{ }_{\tau>0}\right) \approx \frac{1}{1-\theta} \bar{w}_{t}^{1-\theta} \bar{R}^{1-\theta} \mathbb{E} Z_{2}+\bar{w}_{t}^{1-\theta} \bar{R}^{1-\theta}\left(\frac{1+g}{\bar{R}} \mathbb{E} Z_{1}-\mathbb{E} Z_{2}\right) d \tau .
$$

The CEV, denoted by $g_{c}$, is defined by the relationship:

$$
\mathbb{E} u\left(c_{i, 2, t+1_{\tau=0}}\left(1+g_{c}\right)\right)=\mathbb{E} u\left(c_{i, 2, t+1_{\tau>0}}\right),
$$

from which, using the above formulae, we get

$$
\begin{aligned}
\left(1+g_{c}\right)^{1-\theta} \frac{1}{1-\theta} \bar{w}_{t}^{1-\theta} \bar{R}^{1-\theta} \mathbb{E} Z_{2}= & \frac{1}{1-\theta} \bar{w}_{t}^{1-\theta} \bar{R}^{1-\theta} \mathbb{E} Z_{2} \\
& +\bar{w}_{t}^{1-\theta} \bar{R}^{1-\theta}\left(\frac{1+g}{\bar{R}} \mathbb{E} Z_{1}-\mathbb{E} Z_{2}\right) d \tau .
\end{aligned}
$$

Using the respective expressions for $Z_{1}$ and $Z_{2}$ from the proof of Proposition 2, the expression for $g_{c}$ follows.

2. Case $\theta=1$. We have that, evaluated at $\tau=0, \frac{\partial \mathbb{E} u\left(c_{i, 2, t+1}^{\tau, 0}\right)}{\partial \tau}=\frac{1+\lambda}{R} \mathbb{E} Z_{1}-1$. We also have that $\mathbb{E} u\left(c_{i, 2, t+1}^{\tau=0}\right)=\ln \left(\bar{w}_{t} \bar{R}\right)+E \ln \left(\eta_{i, 1, t} \zeta_{t} \varrho_{t+1}\right)$. Therefore:

$$
E u\left(c_{i, 2, t+1}^{\tau>0}\right)=\ln \left(\bar{w}_{t} \bar{R}\right)+E \ln \left(\eta_{i, 1, t} \zeta_{t} \varrho_{t+1}\right)+\left(\frac{1+\lambda}{\bar{R}} E Z_{1}-1\right) d \tau .
$$

For $g_{c}$ we accordingly get $1+g_{c}=\exp \left(\left(\frac{1+\lambda}{R} E Z_{1}-1\right) d \tau\right)$. Using the expression for $Z_{1}$ from the proof of Proposition 2, the expression for $g_{c}$ follows. 
Proof of Proposition 3. As in the proof of Proposition 1, define

$$
R_{t+1}^{p}=\zeta_{t} \bar{R} \tilde{\varrho}_{t+1}+(1+g) \zeta_{t+1} \eta_{i, 2, t+1} \lambda+\tau\left((1+g) \zeta_{t+1}\left(1+\lambda\left(1-\eta_{i, 2, t+1}\right)\right)-\zeta_{t} \bar{R} \varrho_{t+1}\right)
$$

and compute

$$
\begin{aligned}
\left.R_{p, t, t+1}^{-\theta}\right|_{\tau=0} & =\left(\zeta_{t} \bar{R} \varrho_{t+1}+(1+g) \lambda \zeta_{t+1} \eta_{i, 2, t+1}\right)^{-\theta} \\
\left.\frac{\partial R_{p, t, t+1}}{\partial \tau}\right|_{\tau=0} & =(1+g) \zeta_{t+1}\left(1+\lambda\left(1-\eta_{i, 2, t+1}\right)\right)-\zeta_{t} \bar{R} \varrho_{t+1} .
\end{aligned}
$$

From the analogue to condition (26) we then get $\mathbb{E}\left[\frac{(1+g) \zeta_{t+1}\left(1+\lambda\left(1-\eta_{i, 2, t+1}\right)\right)-\zeta_{t} \bar{R} \varrho_{t+1}}{\left(\zeta_{t} \varrho_{t+1}+(1+g) \lambda \zeta_{t+1} \eta_{i, 2, t+1}\right)^{\theta}}\right]>0$, which gives equation (13).

Proof of Proposition 4. First, rewrite (14) as $A_{p e, a m b r}=\mathbb{E}\left[\frac{a Z_{1}-b Z_{2}-1}{1+b Z_{2}}\right]$, where $a \equiv(1+$ $\lambda) \frac{1+g}{\bar{R}}, b \equiv \lambda \frac{1+g}{\bar{R}}$, and $Z_{1} \equiv \frac{\zeta_{t+1}}{\zeta_{t} \varrho_{t+1}}, Z_{2} \equiv \frac{\zeta_{t+1} \eta_{i, 2, t+1}}{\zeta_{t} \varrho_{t+1}}$. Take a second-order Taylor series approximation of the above, around $Z_{2}=Z_{1}=1$ :

$$
\begin{aligned}
A_{p e, a m b r} \approx & \frac{1}{(1+b)^{3}}\left(a b^{2} \mathbb{E}\left[Z_{1} Z_{2}^{2}\right]-3 a b^{2} \mathbb{E}\left[Z_{1} Z_{2}\right]-a b \mathbb{E}\left[Z_{1} Z_{2}\right]+3 a b^{2} \mathbb{E}\left[Z_{1}\right]+\right. \\
& \left.3 a b \mathbb{E}\left[Z_{1}\right]+a \mathbb{E}\left[Z_{1}\right]-(1+b)^{3}\right)
\end{aligned}
$$

Observe that, no interactions are present in term $\mathbb{E} Z_{1}$ and, by Assumption 1 , there are also no interactions in term $\mathbb{E}\left[Z_{1} Z_{2}\right]$. However, observe that

$$
\mathbb{E}\left[Z_{1} Z_{2}^{2}\right]=\mathbb{E}\left[\frac{\zeta_{t+1}}{\zeta_{t} \varrho_{t+1}} \frac{\left(\zeta_{t+1} \eta_{2, i, t+1}\right)^{2}}{\left(\zeta_{t} \varrho_{t+1}\right)^{2}}\right]=\mathbb{E}\left[\zeta_{t+1}^{3}\right] \mathbb{E}\left[\frac{1}{\zeta_{t}^{3}}\right] \mathbb{E}\left[\eta_{2, i, t+1}^{2}\right] \mathbb{E}\left[\frac{1}{\varrho_{t+1}^{3}}\right]
$$

to the effect that an interaction between idiosyncratic and aggregate risk is only present through term $\mathbb{E}\left[Z_{1} Z_{2}^{2}\right]$. We have $\mathbb{E}\left[\eta_{2}^{2}\right]=1+\sigma_{\eta}^{2}, \mathbb{E}\left[\frac{1}{(\varrho)^{3}}\right]=\left(1+\sigma_{\varrho}^{2}\right)^{6}$ and $\mathbb{E} \zeta^{3}=\left(1+\sigma_{\zeta}^{2}\right)^{3}$. Therefore $\mathbb{E}\left[Z_{1} Z_{2}^{2}\right]=\left(1+\sigma_{\zeta}^{2}\right)^{9}\left(1+\sigma_{\varrho}^{2}\right)^{6}\left(1+\sigma_{\eta}^{2}\right)$ and equation (15) immediately follows.

Proof of proposition 5. The proof is by guessing and verifying. As all households are ex-ante identical, we guess for all households $i$ that

$$
s_{i, 2, t+1}=a_{2, t+1}=s(1-\tau) w_{t}=s(1-\tau)(1-\alpha) \Upsilon_{t} \zeta_{t} k_{t}^{\alpha} .
$$


If this is correct, then the equilibrium dynamics are given by

$$
K_{t+1}=a_{2, t+1}=s(1-\tau)(1-\alpha) \Upsilon_{t} \zeta_{t} k_{t}^{\alpha} .
$$

As $k_{t+1}=\frac{K_{t+1}}{\Upsilon_{t+1}(1+\lambda)}$ we get

$$
k_{t+1}=\frac{s(1-\tau)(1-\alpha) \Upsilon_{t} \zeta_{t} k_{t}^{\alpha}}{\Upsilon_{t+1}(1+\lambda)}=\frac{1}{(1+g)(1+\lambda)} s(1-\tau)(1-\alpha) \zeta_{t} k_{t}^{\alpha} .
$$

Recursive substitution gives (17), for any initial capital stock $k_{t-q}$.

To verify (16), notice that our assumptions on savings imply that

$$
c_{i, 1, t}=c_{1, t}=(1-s)(1-\tau)(1-\alpha) \Upsilon_{t} \zeta_{t} k_{t}^{\alpha}
$$

and, by the budget constraint, we have

$$
\begin{aligned}
c_{i, 2, t+1}=s & (1-\tau)(1-\alpha) \Upsilon_{t} \zeta_{t} k_{t}^{\alpha} \alpha \zeta_{t+1} \varrho_{t+1} k_{t+1}^{\alpha-1}+ \\
& +(1-\alpha) \Upsilon_{t+1} \zeta_{t+1} k_{t+1}^{\alpha}\left(\lambda \eta_{i, 2, t+1}+\tau\left(1+\lambda\left(1-\eta_{i, 2, t+1}\right)\right)\right)
\end{aligned}
$$

Using (16) in (29) we get

$$
c_{i, 2, t+1}=\left(\alpha \varrho_{t+1}(1+\lambda)+(1-\alpha)\left(\lambda \eta_{i, 2, t+1}+\tau\left(1+\lambda\left(1-\eta_{i, 2, t+1}\right)\right)\right)\right) \Upsilon_{t+1} \zeta_{t+1} k_{t+1}^{\alpha}
$$

Next, notice that the first-order-condition of household maximization gives

$$
1=\beta \mathbb{E}_{t}\left[\frac{c_{1, t}\left(1+r_{t+1}\right)}{c_{i, 2, t+1}}\right]
$$

Using the above equations for consumption in the two periods, we can rewrite (31) as

$$
\begin{aligned}
1 & =\beta \mathbb{E}_{t}\left[\frac{c_{1, t} \alpha \zeta_{t+1} \varrho_{t+1} k_{t+1}^{\alpha-1}}{\left(\alpha \varrho_{t+1}(1+\lambda)+(1-\alpha)\left(\lambda \eta_{i, 2, t+1}+\tau\left(1+\lambda\left(1-\eta_{i, 2, t+1}\right)\right)\right)\right) \Upsilon_{t+1} \zeta_{t+1} k_{t+1}^{\alpha}}\right] \\
& =\frac{\beta(1-s)}{s} \bar{E}
\end{aligned}
$$

where the last line follows after some transformations and where $\bar{E}$ is defined in equation (19). Equation (18) immediately follows.

Uniqueness is established by convexity of the problem. Given that the solution is unique and given that we have characterized one solution, this is the solution of the 
problem.

As to the upper bound of $\bar{E}$ observe that $\bar{E}=1$ for $\lambda=0$. For $\lambda>0, \bar{E}=\mathbb{E}_{t}\left[\frac{1}{1+x}\right]$ for $x \equiv \frac{1-\alpha}{\alpha(1+\lambda) \varrho_{t+1}}\left(\lambda \eta_{i, 2, t+1}+\tau\left(1+\lambda\left(1-\eta_{i, 2, t+1}\right)\right)\right)$. The assumptions of our model imply that $x \geq 0$, hence $\bar{E}=\mathbb{E}_{t}\left[\frac{1}{1+x}\right] \leq 1$. Finally, observe that the upper bound $\bar{E} \leq 1$ implies the upper bound on the saving rate $s \leq \frac{\beta}{1+\beta}$.

Proof of Proposition 6. 1. Notice from equation (17) that in the ergodic set, i.e., for $q \rightarrow$ $\infty$ we get

$$
k_{t+1}=\left(\frac{1}{(1+g)(1+\lambda)} s(1-\tau)(1-\alpha)\right)^{\frac{1}{1-\alpha}}\left(\prod_{i=0}^{\infty} \zeta_{t-i}^{\alpha^{i}}\right)=k_{m s}\left(\prod_{i=0}^{\infty} \zeta_{t-i}^{\alpha^{i}}\right)
$$

where $k_{m s} \equiv\left(\frac{1}{(1+g)(1+\lambda)} s(1-\tau)(1-\alpha)\right)^{\frac{1}{1-\alpha}}$ is the mean shock equilibrium capital stock, i.e., it is the capital stock that would obtain in equilibrium if nature would draw $\zeta_{t}=1$ in all periods $t-q, \ldots, t$, for $q \rightarrow \infty$.

2. Rewrite (2b) using (3) to make explicit the excess return formulation as in equation (6) to get

$$
\begin{aligned}
c_{2, t+1}=( & s \zeta_{t} \varrho_{t+1} \bar{R}_{t+1}+\lambda \eta_{i, 2, t+1} \frac{\bar{w}_{t+1}}{\bar{w}_{t}}+ \\
& \left.\quad+\tau\left(\left(1+\lambda\left(1-\eta_{i, 2, t+1}\right)\right) \frac{\bar{w}_{t+1}}{\bar{w}_{t}}-s \zeta_{t} \varrho_{t+1} \bar{R}_{t+1}\right)\right) \bar{w}_{t} \zeta_{t+1} .
\end{aligned}
$$

Next, observe that $\bar{w}_{t}=\Upsilon_{t}(1-\alpha) k_{t}^{\alpha}$ and $\bar{R}_{t+1}=\alpha k_{t+1}^{\alpha-1}$ and from step 1 of the proof we have in the ergodic set that

$$
\begin{aligned}
k_{t+1} & =k_{m s}\left(\prod_{i=0}^{\infty} \zeta_{t-i}^{\alpha^{i}}\right)=k_{m s} d(\zeta, t) \text { for } d(\zeta, t) \equiv \prod_{i=0}^{\infty} \zeta_{t-i}^{\alpha^{i}} \\
k_{t} & =k_{m s}\left(\prod_{i=0}^{\infty} \zeta_{t-1-i}^{\alpha^{i}}\right)=k_{m s} d(\zeta, t-1) \text { for } d(\zeta, t-1) \equiv \prod_{i=0}^{\infty} \zeta_{t-1-i}^{\alpha^{i}}
\end{aligned}
$$

Therefore $\bar{w}_{t}=\Upsilon_{t}(1-\alpha) k_{m s}^{\alpha} d(\zeta, t-1)^{\alpha}, \bar{R}_{t+1}=\alpha k_{m s}^{\alpha-1} d(\zeta, t)^{\alpha-1}$ and $\frac{\bar{w}_{t+1}}{\bar{w}_{t}}=(1+$ 
g) $\left(\frac{d(\zeta, t)}{d(\zeta, t-1)}\right)^{\alpha}$. We accordingly get for consumption in $j=1,2$ :

$$
\begin{gathered}
c_{1, t}=(1-s)(1-\tau) \Upsilon_{t} \zeta_{t}(1-\alpha) k_{m s}^{\alpha} d(\zeta, t-1)^{\alpha} \\
c_{2, t+1}=\left(s \zeta_{t} \varrho_{t+1} \alpha k_{m s}^{\alpha-1}+\lambda \eta_{i, 2, t+1}(1+g) \frac{d(\zeta, t)}{d(\zeta, t-1)^{\alpha}}+\right. \\
\left.+\tau\left((1+g)\left(1+\lambda\left(1-\eta_{i, 2, t+1}\right)\right) \frac{d(\zeta, t)}{d(\zeta, t-1)^{\alpha}}-s \zeta_{t} \varrho_{t+1} \alpha k_{m s}^{\alpha-1}\right)\right) \\
\cdot \Upsilon_{t} \zeta_{t+1}(1-\alpha) k_{m s}^{\alpha} d(\zeta, t-1)^{\alpha} d(\zeta, t)^{\alpha-1}
\end{gathered}
$$

To simplify further, notice that $\frac{d(\zeta, t)}{d(\zeta, t-1)^{\alpha}}=\zeta_{t}$. Therefore, second period consumption rewrites as

$$
\begin{aligned}
& c_{2, t+1}=(\left.s \varrho_{t+1} \alpha k_{m s}^{\alpha-1}+\lambda \eta_{i, 2, t+1}(1+g)+\tau\left((1+g)\left(1+\lambda\left(1-\eta_{i, 2, t+1}\right)\right)-s \varrho_{t+1} \alpha k_{m s}^{\alpha-1}\right)\right) \\
& \cdot \Upsilon_{t}(1-\alpha) k_{m s}^{\alpha} \zeta_{t} \zeta_{t+1} d(\zeta, t-1)^{\alpha} d(\zeta, t)^{\alpha-1} .
\end{aligned}
$$

3. Using (33) and (34) in (5), ex-ante utility is accordingly given by

$$
\begin{aligned}
\mathbb{E} u_{t}=\mathbf{c} & +\mathbb{E}[\ln (1-s)+\ln (1-\tau)]+\alpha(1+\beta) \ln k_{m s}+ \\
\beta & \mathbb{E}\left[\operatorname { l n } \left(s \varrho_{t+1} \alpha k_{m s}^{\alpha-1}+\lambda \eta_{i, 2, t+1}(1+g)+\right.\right. \\
& \left.\left.+\tau\left((1+g)\left(1+\lambda\left(1-\eta_{i, 2, t+1}\right)\right)-s \varrho_{t+1} \alpha k_{m s}^{\alpha-1}\right)\right)\right],
\end{aligned}
$$

where $\mathbf{c}$ encompasses all elements that are not affected by $\tau$.

4. Define

$$
B_{1} \equiv \mathbb{E} \frac{\partial \ln (1-s)}{\partial \tau}=-\mathbb{E} \frac{1}{1-s} \frac{\partial s}{\partial \tau}>0
$$

The sign is due to the fact that the partial derivative $\frac{\partial s}{\partial \tau}<0$ as savings are reduced upon introduction of social security (see below). Further define

$$
A_{1} \equiv \mathbb{E} \frac{\partial \ln (1-\tau)}{\partial \tau}=-\left.\frac{1}{1-\tau}\right|_{\tau=0}=-1
$$

As to the implicit return equation for social security, we get, evaluated at $\tau=0$, 
that:

$$
\begin{aligned}
& \frac{\partial \ln \left(s \varrho_{t+1} \alpha k_{m s}^{\alpha-1}+\lambda \eta_{i, 2, t+1}(1+g)+\tau\left((1+g)\left(1+\lambda\left(1-\eta_{i, 2, t+1}\right)\right)-s \varrho_{t+1} \alpha k_{m s}^{\alpha-1}\right)\right)}{\partial \tau} \\
& =\frac{1}{s \varrho_{t+1} \alpha k_{m s}^{\alpha-1}+\lambda \eta_{i, 2, t+1}(1+g)} \cdot . \\
& \quad(\underbrace{(1+g)\left(1+\lambda\left(1-\eta_{i, 2, t+1}\right)\right)-s \varrho_{t+1} \alpha k_{m s}^{\alpha-1}}_{\equiv \tilde{A}_{2}}+\alpha \varrho_{t+1}(\underbrace{\frac{\partial s}{\partial \tau} k_{m s}^{\alpha-1}}_{\equiv \tilde{B}_{2}}+\underbrace{s(\alpha-1) k_{m s}^{\alpha-2} \frac{\partial k_{m s}}{\partial \tau}}_{\equiv \tilde{C}})) .
\end{aligned}
$$

First, look at

$$
\frac{1}{s \varrho_{t+1} \alpha k_{m s}^{\alpha-1}+\lambda \eta_{i, 2, t+1}(1+g)} \tilde{A}_{2}=\frac{\frac{1-\alpha}{\alpha(1+\lambda)} \frac{1+\lambda\left(1-\eta_{i, 2, t+1}\right)}{\varrho_{t+1}}-1}{1+\frac{(1-\alpha) \lambda}{\alpha(1+\lambda)} \frac{\eta_{i, 2, t+1}}{\varrho_{t+1}}} .
$$

Multiplying the above term by $\beta$ - cf. equation (35) - , taking expectations and subtracting -1 in order to acknowledge the effects of taxation on income (from equation (37)), gives term $A$.

Next, look at

$$
\frac{\alpha \varrho_{t+1}}{s \varrho_{t+1} \alpha k_{m s}^{\alpha-1}+\lambda \eta_{i, 2, t+1}(1+g)} \tilde{B}_{2}=\frac{\partial s}{\partial \tau} \frac{1}{s} \frac{1}{1+\frac{(1-\alpha) \lambda}{\alpha(1+\lambda)} \frac{\eta_{i, 2, t+1}}{\varrho_{t+1}}}<0 .
$$

Multiplying the above by $\beta$ - cf. equation (35) - , taking expectations and combining the resulting term with equation (36), we get

$$
\begin{aligned}
B & \equiv \mathbb{E}\left[\frac{\partial s}{\partial \tau}\left(\beta \frac{1}{s} \frac{1}{1+\frac{(1-\alpha) \lambda}{\alpha(1+\lambda)} \frac{\eta_{i, 2, t+1}}{\varrho_{t+1}}}-\frac{1}{1-s}\right)\right] \\
& =\left.\beta \epsilon_{s, \tau}\right|_{\tau=0}\left(\left.\bar{E}\right|_{\tau=0}-\left.\bar{E}\right|_{\tau=0}\right)=0
\end{aligned}
$$

where $\left.\epsilon_{s, \tau}\right|_{\tau=0}=\frac{\partial s}{\partial \tau} \frac{1}{s}<0$ is the semi-elasticity of the saving rate in $\tau$, evaluated at $\tau=0$. It is given by

$$
\left.\left.\epsilon_{s, \tau}\right|_{\tau=0} \equiv \frac{\partial s}{\partial \tau} \frac{1}{s}\right|_{\tau=0}=-\left.\beta(1-s)^{2} \frac{1}{s}\right|_{\tau=0}=-\frac{\left.\overline{\bar{E}}\right|_{\tau=0}}{\left.\left(1+\left.\beta \bar{E}\right|_{\tau=0}\right) \bar{E}\right|_{\tau=0}}
$$


where $\left.\overline{\bar{E}}\right|_{\tau=0} \equiv-\left.\frac{\partial \bar{E}}{\partial \tau}\right|_{\tau=0}=\mathbb{E}_{t}\left[\frac{\frac{1-\alpha}{\alpha(1+\lambda)} \frac{1+\lambda\left(1-\eta_{i, 2, t+1}\right)}{\varrho_{t+1}}}{\left(1+\frac{(1-\alpha) \lambda}{\alpha(1+\lambda)} \frac{\eta_{i, 2, t+1}}{\varrho_{t+1}}\right)^{2}}\right]>0$.

Finally, look at

$$
\frac{\alpha \varrho_{t+1}}{s \varrho_{t+1} \alpha k_{m s}^{\alpha-1}+\lambda \eta_{i, 2, t+1}(1+g)} \tilde{C}=-(1-\alpha) \frac{1}{1+\frac{(1-\alpha) \lambda}{\alpha(1+\lambda)} \frac{\eta_{i, 2, t+1}}{\varrho t+1}} \frac{\partial \ln k_{m s}}{\partial \tau} .
$$

Multiplying the above by $\beta$, taking expectations and combining it with equation (35), all terms incorporating $\frac{\partial \ln k_{m s}}{\partial \tau}$ are given by

$$
C=\left(\alpha(1+\beta)-\left.\beta(1-\alpha) \bar{E}\right|_{\tau=0}\right) \mathbb{E}\left[\frac{\partial \ln k_{m s}}{\partial \tau}\right]
$$

Turning to $\frac{\partial \ln k_{m s}}{\partial \tau}$ we find that, at $\tau=0$, we have

$$
\frac{\partial \ln k_{m s}}{\partial \tau}=\frac{1}{1-\alpha}\left(\frac{\partial \ln s}{\partial \tau}+\frac{\partial \ln (1-\tau)}{\partial \tau}\right)=-\frac{1}{1-\alpha}\left(1-\left.\epsilon_{s, \tau}\right|_{\tau=0}\right)<0
$$

where the sign follows from the fact that $\left.\epsilon_{s, \tau}\right|_{\tau=0} \in(0,1)$. The expression for term $C$ given in the proposition then follows.

Proof of Proposition 7 and Lemma 1. From the aggregate resource constraint in our model with $\delta=1, N_{j, t}=1, j=1,2$, we get $c_{1, t}+c_{2, t}+K_{t+1}=F\left(K_{t}, \Upsilon_{t} L_{t}\right)$ and by homogeneity of $F(\cdot, \cdot)$ maximizing per capita consumption $\bar{c}=\frac{c_{1, t}+c_{2, t}}{2}$ is equivalent to maximizing

$$
\max \left\{\frac{F\left(K_{t}, \Upsilon_{t} L_{t}\right)}{N_{t}}-\frac{K_{t+1}}{N_{t}}\right\}
$$

As $N_{t}=N_{t+1}=2, N_{1, t}=N_{2, t}=1, L_{t}=1+\lambda$ and recalling that $k_{t}=\frac{K_{t}}{\Upsilon_{t} L_{t}}$ we have that $\frac{K_{t+1}}{N_{t+1}}=k_{t+1} \Upsilon_{t+1}(1+\lambda) \frac{1}{2}$ and $\frac{L_{t}}{N_{t}}=(1+\lambda) \frac{1}{2}$. Maximizing (39) in steady state where $k_{t+1}=k_{t}=k$ is equivalent to $\max \{f(k)-(1+g) k\}$. Using that $\left.f(k)\right)=k^{\alpha}$ we get the golden rule capital stock $k_{G R}=\left(\frac{\alpha}{1+g}\right)^{\frac{1}{1-\alpha}}$.

From equation (16) we get that the steady state capital stock in the deterministic $\lambda=0$ economy is $k=\left(\frac{\beta(1-\alpha)}{(1+\beta)(1+g)}\right)^{\frac{1}{1-\alpha}}$. Hence the deterministic $\lambda=0$ economy is dynamically efficient iff $\frac{\beta}{1+\beta}<\frac{\alpha}{1-\alpha}$.

Finally, observe that $C<$ iff $\alpha(1+\beta)-\left.\beta(1-\alpha) \bar{E}\right|_{\tau=0}>0$ which we can rewrite 
to $\frac{\left.\beta \bar{E}\right|_{\tau=0}}{1+\beta}<\frac{\alpha}{1-\alpha}$. Dynamic efficiency of the mean shock economy is a sufficient condition because from $0<\left.\bar{E}\right|_{\tau=0} \leq 1$, we obviously have that $\frac{\left.\beta \bar{E}\right|_{\tau=0}}{1+\beta} \leq \frac{\beta}{1+\beta}$, and therefore $\frac{\left.\beta \bar{E}\right|_{\tau=0}}{1+\beta}<\frac{\alpha}{1-\alpha}$.

Proof of proposition 8. 1. The partial derivative of term $A$ immediately follows by setting to zero $\sigma_{\zeta}^{2}$ in term $\frac{\left.\partial A_{p e, a m b r}\right|_{\theta=1}}{\partial \sigma_{\eta}^{2}}$, cf. the (proof of) Proposition 4.

2. The partial derivative of term $C$ is given by

$$
\frac{\partial C}{\partial \sigma_{\eta}^{2}}=\underbrace{\left(\alpha(1+\beta)-\left.\beta(1-\alpha) \overline{\bar{E}}\right|_{\tau=0}\right)}_{>0} \frac{1}{1-\alpha} \underbrace{\frac{\left.\partial \epsilon_{s, \tau}\right|_{\tau=0}}{\partial \sigma_{\eta}^{2}}}_{<0}+\beta \underbrace{\left(1-\left.\epsilon_{s, \tau}\right|_{\tau=0}\right)}_{>0} \underbrace{\frac{\left.\partial \overline{\bar{E}}\right|_{\tau=0}}{\partial \sigma_{\eta}^{2}}}_{>0}
$$

where it remains to establish that, indeed, $\frac{\left.\partial \overline{\bar{E}}\right|_{\tau=0}}{\partial \sigma_{\eta}^{2}}>0$ and $\frac{\left.\partial \epsilon_{s, \tau}\right|_{\tau=0}}{\partial \sigma_{\eta}^{2}}<0$ :

(a) To evaluate $\frac{\left.\partial \bar{E}\right|_{\tau=0}}{\partial \sigma_{\eta}^{2}}$, approximate $\left.\bar{E}\right|_{\tau=0}$ to get

$$
\left.\bar{E}\right|_{\tau=0} \approx\left[\frac{a^{2} \mathbb{E} Z_{3}^{2}-\left(3 a^{2}+a\right) \mathbb{E} Z_{3}+3 a^{2}+3 a+1}{a^{3}+3 a^{2}+3 a+1}\right]
$$

With $\mathbb{E}\left[Z_{3}\right]=\left(1+\sigma_{\varrho}^{2}\right)$ and $\mathbb{E}\left[Z_{3}^{2}\right]=\left(1+\sigma_{\eta}^{2}\right)\left(1+\sigma_{\varrho}^{2}\right)^{3}$ equation (24) follows.

(b) As to the partial derivative of $\left.\epsilon_{s, \tau}\right|_{\tau=0}$ with respect to $\sigma_{\eta}^{2}$, recall equation (38).

To determine how $\left.\epsilon_{s, \tau}\right|_{\tau=0}$ reacts to changes in $\sigma_{\eta}^{2}$ it remains to determine $\frac{\left.\partial \overline{\bar{E}}\right|_{\tau=0}}{\partial \sigma_{\eta}^{2}}$.

Take a second-order Taylor series expansion of $\left.\overline{\bar{E}}\right|_{\tau=0}$ to get

$$
\begin{aligned}
\left.\overline{\bar{E}}\right|_{\tau=0} \approx & \frac{1}{(1+a)^{4}}\left[\left(3 a^{2} b \mathbb{E} Z_{3}^{2}-\left(8 a^{2}+2 a\right) b \mathbb{E} Z_{3}+\left(6 a^{2}+4 a+1\right) b\right) \mathbb{E} Z_{4}+\right. \\
& \left.\left(2 a^{2}-a^{3}\right) \mathbb{E} Z_{3}^{2}+\left(3 a^{3}-4 a^{2}-a\right) \mathbb{E} Z_{3}-3 a^{3}\right] .
\end{aligned}
$$

To evaluate this expression under log-normality observe that $\mathbb{E}\left[Z_{4}\right]=\mathbb{E}\left[\frac{1}{\left(\varrho_{t+1}\right)}\right]=$ $1+\sigma_{\varrho}^{2}$. Furthermore we have that

$$
\mathbb{E}\left[Z_{3}^{2} Z_{4}\right]=\mathbb{E}\left[\frac{\eta_{t+1}^{2}}{\left(\varrho_{t+1}\right)^{3}}\right]=\mathbb{E} \eta_{t+1}^{2} \mathbb{E}\left[\frac{1}{\left(\varrho_{t+1}\right)^{3}}\right]
$$

We have that $\mathbb{E} \eta_{2}^{2}=1+\sigma_{\eta}^{2}$ and $\mathbb{E}\left[\frac{1}{\left(\varrho_{t+1}\right)^{3}}\right]=\left(1+\sigma_{\varrho}^{2}\right)^{6}$. Therefore $\mathbb{E}\left[Z_{3}^{2} Z_{4}\right]=$ 
$\left(1+\sigma_{\eta}^{2}\right)\left(1+\sigma_{\varrho}^{2}\right)^{6}$. The next term is $\mathbb{E}\left[Z_{3} Z_{4}\right]=\mathbb{E}\left[\frac{\eta_{t+1}}{\left(\varrho_{t+1}\right)^{2}}\right]=\left(1+\sigma_{\varrho}^{2}\right)^{3}$. Finally $\mathbb{E}\left[Z_{4}\right]=\mathbb{E}\left[\frac{1}{\left(\varrho_{t+1}\right)}\right]=1+\sigma_{\varrho}^{2}$. Consequently,

$$
-\frac{\left.\partial \overline{\bar{E}}\right|_{\tau=0}}{\partial \sigma_{\eta}^{2}}=-\left(\sigma_{\varrho}^{2}+1\right)^{3} \frac{3 a^{2} b\left(\sigma_{\varrho}^{2}+1\right)^{3}-a^{3}+2 a^{2}}{(a+1)^{4}}<0 .
$$

The negative sign follows from the fact that

$$
3 a^{2} b\left(\sigma_{\varrho}^{2}+1\right)^{3}-a^{3}+2 a^{2}>3 a^{2} b-a^{3}+2 a^{2}>0
$$

because

$$
\begin{aligned}
& 3 a^{2} b-a^{3}+2 a^{2}>0 \\
& \Leftrightarrow \quad(3-\alpha)(1+\lambda)>(1-\alpha) \lambda
\end{aligned}
$$

and the latter holds for any $\alpha \in(0,1)$ and $\lambda \in(0,1)$ because $(3-\alpha)(1+\lambda)>1$ and $(1-\alpha) \lambda<1$. Therefore, as $\frac{\left.\partial \bar{E}\right|_{\tau=0}}{\partial \sigma_{\eta}^{2}}>0$ and $\frac{\left.\partial \overline{\bar{E}}\right|_{\tau=0}}{\partial \sigma_{\eta}^{2}}<0$ we have $\frac{\left.\partial \epsilon_{s, \tau}\right|_{\tau=0}}{\partial \sigma_{\eta}^{2}}<$ 0 . 


\section{B Supplementary Appendix: Additional Material}

\section{B.1 Comparison to Three-Generations Model}

The purpose of this subsection is to illustrate the quasi-formal equivalence of the twoperiod model with a subperiod structure to a three-period model. To this aim, consider a setting like in Subsection 3.2. Households work in the first two periods of life and are retired in the third. There is no idiosyncratic risk in the first period. We again assume that households only care about consumption in retirement (third period). Third period consumption is then

$$
\begin{aligned}
c_{i, 3, t+2} & =w_{t}(1-\tau) R_{t+1} R_{t+2}+w_{t+1} \eta_{i, 2, t+1}(1-\tau) R_{t+2}+2 \tau w_{t+1} \\
& =\bar{w}_{t}\left(\zeta_{t} \bar{R}^{2} \varrho_{t+1} \varrho_{t+2}+(1+g) \zeta_{t+1} \eta_{i, 2, t+1} \bar{R} \varrho_{t+2}+\right. \\
& \left.+\quad \tau\left(2(1+g)^{2} \zeta_{t+2}-\left(\zeta_{t} \bar{R}^{2} \varrho_{t+1} \varrho_{t+2}+(1+g) \zeta_{t+1} \eta_{i, 2, t+1} \bar{R} \varrho_{t+2}\right)\right)\right) .
\end{aligned}
$$

To interpret this in light of the analysis in subsection 3.2, again consider the case where $\tau=0$ and $\bar{w}_{t}=1$. Then

$$
c_{i, 3, t+2}=\bar{R}^{2} \underbrace{\zeta_{t} \varrho_{t+1} \varrho_{t+2}}_{\equiv \psi}+(1+g) \bar{R}+(1+g) \bar{R}(\underbrace{\zeta_{t+1} \eta_{i, 2, t+1} \varrho_{t+2}}_{\equiv \phi}-1) .
$$

Observe that this is not a situation with mean zero independent "background" risk because of aggregate return risk in the second period: Term $\rho_{t+2}$ shows up in both random variables $\psi$ and $\phi$ to the effect that $\psi$ and $\phi$ are not independent.

Therefore, we additionally assume, somewhat artificially, that households only have access to a risk-free saving technology in the second period of life. We then get

$$
c_{i, 3, t+2}=\bar{R}^{2} \underbrace{\zeta_{t} \varrho_{t+1}}_{\equiv \tilde{\psi}}+(1+g) \bar{R}+(1+g) \bar{R}(\underbrace{\zeta_{t+1} \eta_{i, 2, t+1}}_{\equiv \tilde{\phi}}-1) .
$$

This is formally equivalent to equation (12), i.e., we are back at a situation with independent additive "background" risk, again with the multiplicative interaction of risks via $\zeta_{t+1}$ in term $\tilde{\phi}$.

Remark 1. In fact, we can develop this further to a multi-period model. Suppose a household born in period $t$ works for $j_{r}$ years, saves his labor income in each of these 
years and is retired in period $j_{r}+1$, consuming all these resources. We now allow for an idiosyncratic wage shock in each period (also in the first). Consumption at age $j_{r}+1$, period $t+j_{r}$, then reads as

$$
\begin{gathered}
c_{i, j_{r}+1, t+j_{r}}=(1-\tau)\left(w_{t} \eta_{i, 1, t} \prod_{s=t+1}^{t+j_{r}} R_{s}+w_{t+1} \eta_{i, 2, t+1} \prod_{s=t+2}^{t+j_{r}} R_{s}+\ldots+w_{t+j_{r}-1} \eta_{i, j_{r}, t+j_{r}-1} R_{t+j_{r}}\right. \\
+j_{r} \tau w_{t+j_{r}}
\end{gathered}
$$

Now let $\eta$ obey a random walk in logs as we assumed in our illustrative back of the envelope calculation of Section 3, i.e.,

$$
\eta_{i, j, t}=\eta_{i, j-1, t-1} \epsilon_{j, t}, \quad \eta_{i, 1, t}=\epsilon_{1, t}
$$

and assume that the $\log$ of $\zeta_{t}$ is an auto-correlated process with correlation coefficient $\rho$. This is roughly consistent with our earlier approximation of zero dependence over long horizons. I.e., assume that

$$
\zeta_{t}=\zeta_{t-1}^{\rho} \nu_{t}
$$

We then get

$$
\begin{array}{r}
c_{i, j r+1, t+j r}=w(1-\tau) \cdot\left(\sum_{j=1}^{j_{r}} \bar{R}^{j_{r}-j+1} \cdot \zeta_{t-1}^{\rho^{j}}\left(\prod_{\ell=1}^{j} \nu_{t+j-\ell}^{\rho^{\ell-1}}\right) \cdot\left(\prod_{\ell=1}^{j} \epsilon_{j-\ell+1, t+j-\ell}\right)\right. \\
\left.\cdot\left(\prod_{s=t+j}^{t+j_{r}} \varrho_{s}\right)\right) \\
+j_{r} \tau w_{t+j_{r} .}
\end{array}
$$

This establishes that, in a more realistic (yet of course still stylized) example, numerous multiplicative and additive interactions are present.

\section{B.2 Supplementary Appendix: Intuition Behind Proposition 6}

This subsection explains why in Proposition 6 , term $B=0$, and why the aggregate productivity shock, $\zeta_{t}$, drops out. Observe that the consumption equations in general equilibrium, as derived in the proof of Proposition 5 are given by

$$
\begin{aligned}
& c_{1, t}=(1-s(\tau))(1-\tau)(1-\alpha) \Upsilon_{t} \zeta_{t} k_{t}^{\alpha} \\
& c_{2, t}=\left(\alpha \varrho_{t+1}(1+\lambda)+(1-\alpha)\left(\lambda \eta_{i, 2, t+1}+\tau\left(1+\lambda\left(1-\eta_{i, 2, t+1}\right)\right)\right)\right) \Upsilon_{t+1} \zeta_{t+1} k_{t+1}^{\alpha}
\end{aligned}
$$


Combining the above with (17) and using the resulting term in the expected utility function we get

$$
\begin{gathered}
U_{t-1}=\Gamma+\ln (1-s(\tau))+\beta \ln (s(\tau))+\alpha(1+\alpha \beta) \ln \left(k_{t}\right)+ \\
+\ln (1-\tau)(1+\beta)+\beta \underbrace{\mathbb{E}_{t-1}\left[\ln \left(\alpha \varrho_{t+1}(1+\lambda)+(1-\alpha)\left(\lambda \eta_{i, 2, t+1}+\tau\left(1+\lambda\left(1-\eta_{i, 2, t+1}\right)\right)\right)\right)\right]}_{=\Psi(\tau)} \\
+\underbrace{\mathbb{E}_{t-1}\left[\ln \left(\zeta_{t}\right)(1+\beta)+\beta \ln \left(\zeta_{t+1}\right)\right]}_{=\Phi}
\end{gathered}
$$

where $\Gamma$ collects exogenous parameters that do not depend on $\tau$ and do not contain any risk terms. The equation allows us to establish three important insights, which will help understand proposition 6. First, welfare costs from aggregate technology fluctuations as reflected in term $\Phi$ are purely additive and neither affected by policy nor by precautionary savings behavior. Second, interactions between idiosyncratic and aggregate risk are reflected in term $\Psi(\tau)$. Note that only aggregate return risk shows up. This term captures the welfare losses from fluctuations of the idiosyncratic labor income component and the aggregate return. While these welfare losses can be affected by policy - to make this explicit, $\Psi$ is written as a function of $\tau$-, the saving rate does not enter this term. From these observations we can conclude that general equilibrium price reactions - through the adjustment of the aggregate capital stock - fully offset any utility enhancing effects of precautionary savings. This is true not only in the long-run equilibrium but in each period. While households experience utility gains from self-insurance against fluctuations in partial equilibrium, these utility gains from self-insurance vanish to exist in general equilibrium. The reason is the well-known capital externality typical to Aiyagari (1995) models: In response to the increase of precautionary savings the capital stock increases with adverse welfare consequences. In the specific model considered here, these general equilibrium reactions exactly offset utility gains from precautionary savings. As a consequence, when social security is introduced and provides insurance, there will not be any utility gains from reductions of precautionary savings.

\section{B.3 Welfare in General Equilibrium for $\lambda=0$}

This subsection derives the welfare effects in general equilibrium for the special case where $\lambda=0$, because in this case, a very intuitive condition obtains. That is, we start from Proposition 6 and shut down all effects of idiosyncratic risk. To interpret term $A$, 
notice that for $\lambda=0$ we can write

$$
\frac{A}{1+\beta}=\frac{(1-\alpha) \beta}{\alpha(1+\beta)} \mathbb{E}\left[\frac{1}{\varrho_{t+1}}\right]-1 .
$$

To further interpret this term, consider an artificial economy, namely the steady state of the mean-shock equilibrium (MSE) of the economy.

Definition 1. In the mean-shock equilibrium, equilibrium dynamics are characterized by equation (16) but nature always draws the mean of aggregate shocks, i.e., $\zeta_{t}=\varrho_{t}=1$ for all $t$.

From this definition the steady state mean shock equilibrium capital stock in the $\lambda=0$, $\tau=0$ economy, denoted by $k_{m s}$, is given by

$$
k_{m s}=\left(\frac{(1-\alpha) \beta}{(1+\beta)(1+g)}\right)^{\frac{1}{1-\alpha}} .
$$

Consequently, the mean shock expected gross return is $R_{m s}=\alpha k_{m s}^{\alpha-1}=(1+g) \frac{\alpha}{1-\alpha} \frac{1+\beta}{\beta}$. Hence, in this mean shock equilibrium, term $A>0$ iff

$$
\frac{1+g}{R_{m s}} \mathbb{E}\left[\frac{1}{\varrho_{t+1}}\right]-1>0 .
$$

This means that the risk adjusted return of social security has to exceed the rental rate in the mean shock equilibrium. A mean preserving spread of $\varrho_{t+1}$ increases $A$ because insurance becomes more valuable. This establishes the analogy to our earlier interpretation of term $A_{p e, a b r}$ in Subsection 3.2 for an economy without any idiosyncratic risk. 


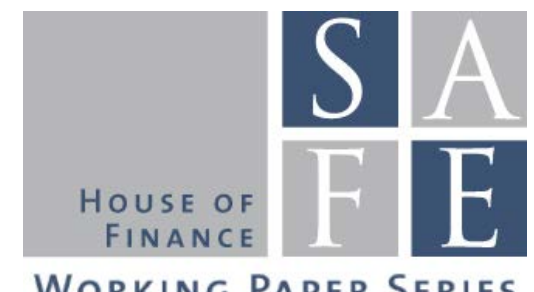

Working PAPER SERIES

\section{Recent Issues}

No. 70 Deyan Radev

No. 69 Nina Biljanovska, Spyros Palligkinis

No. 68 Tobias Tröger

No. 67 Sascha Baghestanian, Paul J. Gortner, Joel van der Weele

No. 66 Tobias Tröger

No. 65 Elia Berdin, Helmut Gründl

No.64 Daniel Herbold

No. 63 Nicola Fuchs-Schündeln, Michael Haliassos

No. 62 Patrick Behr, Alejandro H. Drexler, Reint Gropp, Andre Guettler

No. 61 Iñaki Aldasoro, Mike Seiferling

No. 60 Stefano Colonnello, Giuliano Curatola, Ngoc Giang Hoang

No. 59 Daniel Harenberg, Alexander Ludwig

No. 58 Michael Haliassos, Thomas Jansson, Yigitcan Karabulut

No. 57 Brigitte Haar
Assessing Systemic Fragility - a Probabilistic Perspective

Control Thyself: Self-Control Failure and Household Wealth

How Special Are They? - Targeting Systemic Risk by Regulating Shadow Banking

Peer Effects and Risk Sharing in Experimental Asset Markets

\section{Corporate Groups}

The Effects of a Low Interest Rate Environment on Life Insurers

A Repeated Principal-Agent Model with Onthe-Job Search

Does Product Familiarity Matter for Participation?

Financial Incentives and Loan Officers Behavior: Multitasking and Allocation of Effort Under an Incomplete Contract

Vertical Fiscal Imbalances and the Accumulation of Government Debt

Executive Compensation Structure and Credit Spreads

Social Security and the Interactions Between Aggregate and Idiosyncratic Risk

Incompatible European Partners? Cultural Predispositions and Household Financial Behavior

Financial Regulation in the EU - Cross-Border Capital Flows, Systemic Risk and the European Banking Union as Reference Points for EU Financial Market Integration 